\title{
Conflict Analysis for Cooperative Maneuvering with Status and Intent Sharing via V2X Communication
}

\author{
Hao M. Wang, Sergei S. Avedisov, Tamás G. Molnár, Ahmed Hamdi Sakr, Onur Altintas, and Gábor Orosz
}

\begin{abstract}
In this paper, we establish the concept of conflict analysis and demonstrate its applicability to aid the decision making of vehicles at different levels of automation and cooperation. In particular, we assume that the participating vehicles are equipped with vehicle-to-everything (V2X) communication and study cooperative maneuvers under status sharing and intent sharing. Conflict-free maneuvering strategies are developed and communication requirements for such strategies are determined. To demonstrate the developed framework, we investigate a scenario with an ego vehicle merging to a main road while another vehicle is approaching on that road. The results are demonstrated by both experimental data using real vehicles on a test track and simulations based on real highway data.
\end{abstract}

Index Terms-V2X communication, conflict analysis, connected and automated vehicles.

\section{INTRODUCTION}

$\mathbf{C}$ ONFLICT may arise between different road participants when their future trajectories cross each other. These conflicts, when not managed appropriately, may lead to safety hazards and eventually cause accidents. Human drivers use many cues from other road users to prevent conflicts and there is a clear need for automated vehicles to possess similar qualities in versatile road scenarios. Vehicle-to-everything (V2X) communication can be the enabling technology that allows vehicles of different levels of automation to manage conflicts while utilizing different levels of cooperation [1], [2].

Prior results show that V2X communication may be utilized by automated vehicles in order to improve their performance according to multiple metrics while performing different maneuvers [3]. During the next few decades, one may expect to deal with mixed traffic scenarios consisting of human-driven vehicles and vehicles of different automation levels [4], [5]. This opens up new opportunities for cooperation between different road participants as summarized in the top panel

Hao Wang and Gábor Orosz are with the Department of Mechanical Engineering, University of Michigan, Ann Arbor, MI 48109, USA \{ haowangm, orosz\}@umich.edu.

Sergei Avedisov and Onur Altintas are with Toyota Motor North America R\&D - InfoTech Labs, Mountain View, CA 94043, USA \{sergei.avedisov, onur.altintas\}etoyota.com.

Tamás Molnár is currently with the Department of Mechanical and Civil Engineering, California Institute of Technology, Pasadena, CA 91125, USA tmolnarecaltech.edu. He was with the Department of Mechanical Engineering, University of Michigan, Ann Arbor, MI 48109, USA during the time when the results were generated.

Ahmed Hamdi Sakr is currently with the Department of Electrical and Computer Engineering, University of Windsor, Windsor, ON N9B 3P4, Canada. ahmed.sakr@uwindsor.ca. He was with Toyota Motor North America R\&D - InfoTech Labs, Mountain View, CA 94043, USA during the time when the results were generated.

Gábor Orosz is also with the Department of Civil and Environmental Engineering, University of Michigan, Ann Arbor, MI 48109, USA.
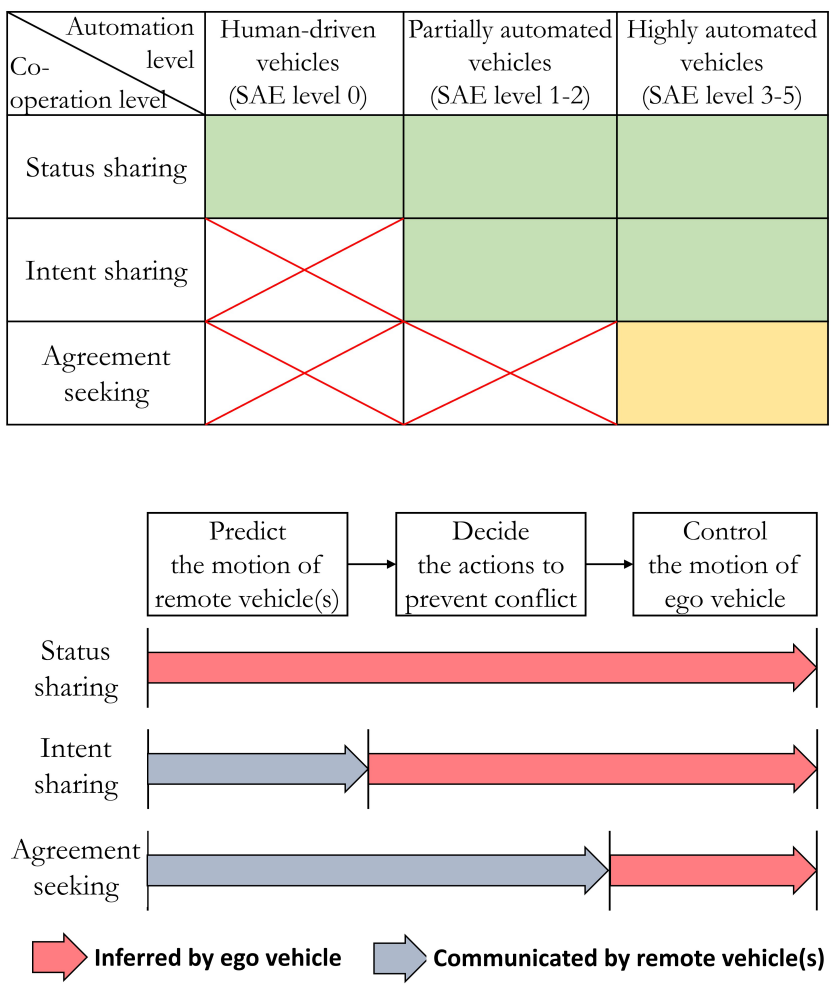

Fig. 1. Top: Levels of automation and cooperation targeted in this paper (green shading). Bottom: Conflict prevention performed by a connected automated vehicle under different cooperation levels.

of Fig. 1. Most prior research efforts focused on the yellow shaded part where highly automated vehicles use maneuver coordination messages to reach agreements on future maneuvers [6]-[9]. Instead, in this paper, we focus on the green shaded part where vehicles of different automation levels cooperate to prevent conflicts. First, we establish conflict analysis for scenarios where vehicles share their current status (e.g., position and velocity) via V2X communication and the participating vehicles include connected human-driven vehicles (CHVs) as well as connected automated vehicles (CAVs). We analyze the situation from the perspective of an ego vehicle that is considered to be a CAV. Then we extend conflict analysis to cases where vehicles share their intent regarding their future motion and quantify the benefits of such information from the perspective of the ego vehicle.

We utilize the results of conflict analysis to aid the decision making of the ego vehicle (CAV) as illustrated at the bottom panel of Fig. 1. The CAV shall predict the motion of remote vehicles based on the available information and use these pre- 
dictions when deciding what action to take so that no conflicts arise. Then the ego vehicle shall execute the chosen action using a control algorithm. When the CAV receives information only about the current status of the remote vehicles, the decisions that ensure conflict-free maneuvers are expected to be conservative. We will show that performance can be improved when the remote vehicles also provide information about their intent (which requires them to have at least some low level of automation). In scenarios when vehicles of higher levels of automation meet, they may agree about what actions to take in a cooperative manner and further reduce conservatism [6]-[9]. There also exists a sizable literature of strategies where even the control actions of the highly automated vehicles are carried out cooperatively. Such strategies include virtual platooning [10], optimal control [11]-[13], model predictive control [14], and reachability analysis [15]-[18]. Applications include not only automated ground vehicles but also unmanned aerial vehicles [19], [20]. Such strategies can significantly increase efficiency but also require a high level of automation of all traffic participants and are outside of the scope of this article.

Managing conflicts in mixed autonomous systems generated interests in multiple research communities. In [21] a framework for robot-human collision avoidance was proposed, where prediction of human behavior was achieved by a statistical model without considering interaction, and robot trajectories generated by sequential planning were tracked by robust reachability-based controllers. In [22], game theory was used to model human decision making, and reinforcement learning was used for intelligent agents to generate the optimal action sequence. Recent studies on conflict resolutions between automated and human-driven vehicles, especially in scenarios such as merges, lane changes and roundabouts [23]-[25], used a variety of optimal control techniques including dynamic programming, model predictive control, and reinforcement learning. However, these methods suffer from limited scalability due to the "curse of dimensionality" arising in dynamic optimization problems. Moreover, to the best of our knowledge, no prior works studied the effects of wireless communication (e.g., communication rate, range, and information types) on conflict resolution in mixed-autonomous vehicle scenarios. This paper provides a first effort to fill these gaps by building a scalable framework of conflict analysis for vehicles of various levels of automation and cooperation, while unveiling the benefits of V2X connectivity.

We define a conflict as an event when the trajectories of traffic participants intersect so that they appear at the same location at the same time. Since vehicles have finite dimensions, we define a conflict zone of a finite size. For simplicity, we focus on cooperative maneuvers where the conflict zone is fixed to the ground as depicted in Fig. 2(a)(c). Then, to prevent conflicts, no more than one vehicle must be present in the conflict zone at the same time. We develop conflict analysis using the representative example where the ego vehicle merges to a main road while a remote vehicle is approaching on that road [26]. However, the methods we develop can be applied to a larger set of maneuvers where multiple vehicles must enter a given spatial domain. Examples include intersections, roundabouts, and lane changes [11],
[27], [28] with conflict zones of different shapes and sizes. We consider conflict prevention from the perspective of the ego vehicle which, according to the traffic rules, must yield to the other vehicle approaching the conflict zone on the main road. It is assumed that both vehicles are equipped with V2X communication devices and the ego vehicle is automated, thus, referred to as a CAV; see Fig. 2(d). We demonstrate how conflict analysis can be utilized by the CAV for decision making under different levels of cooperation. Namely, we consider status sharing and intent sharing scenarios which can be supported by different types of wireless messages [1].

In conflict analysis, we consider the performance limits of both vehicles and calculate the so-called no-conflict, conflict, and uncertain domains in the state space for the cases when the ego vehicle merges ahead of the remote vehicle and when it merges behind. We demonstrate that the corresponding conflict charts can be used for decision making by the ego vehicle. Based on the conflict analysis, we also derive a $\mathrm{V} 2 \mathrm{X}$ communication range requirement. If the ego vehicle receives status sharing messages before the remote vehicle enters this range, conflict-free maneuvers can be guaranteed independent of the remote vehicle's future motion. Then, we design a longitudinal controller for the ego CAV to execute the maneuver. We demonstrate that even a single status message from the remote vehicle can enable the vehicle to prevent conflicts and that the time efficiency can be significantly improved when receiving more status updates. We also show that by receiving intent information from the remote vehicle, a CAV can significantly enhance its capability of preventing conflicts and also improve its time efficiency. The developed conflict analysis framework is demonstrated by real vehicles via experiments performed at a test track and via simulations using highway traffic data collected at South-East Michigan.

This paper extends and generalizes the initial results presented in the conference paper [29], where conflict analysis was initially proposed considering status sharing between vehicles. The major technical contributions of this paper are summarized as follows. (i) We build a generalized and scalable framework for conflict analysis incorporating different levels of cooperation, which enables fast and reliable decision making and controller design. (ii) We systematically study and quantify the benefits of status and intent sharing via V2X communication in conflict resolution. (iii) We demonstrate the applicability of the framework experimentally using real vehicles and real traffic data.

The rest of the paper is organized as follows. In Sec. II the mathematical model for vehicle dynamics is constructed. In Sec. III we establish the conflict analysis framework. We introduce the concept of conflict charts, derive the V2X communication range requirement, and use these results to construct a decision making rule for the ego vehicle. In Sec. IV we investigate the scenario when the remote vehicle shares its intent with the ego vehicle via V2X communication. In Sec. V we design a controller that enables the ego CAV to execute non-conflicting maneuvers. In Sec. VI experiments performed at a test track and simulations using real traffic data are presented. Finally, in Sec. VII we conclude the paper and point out future research directions. 


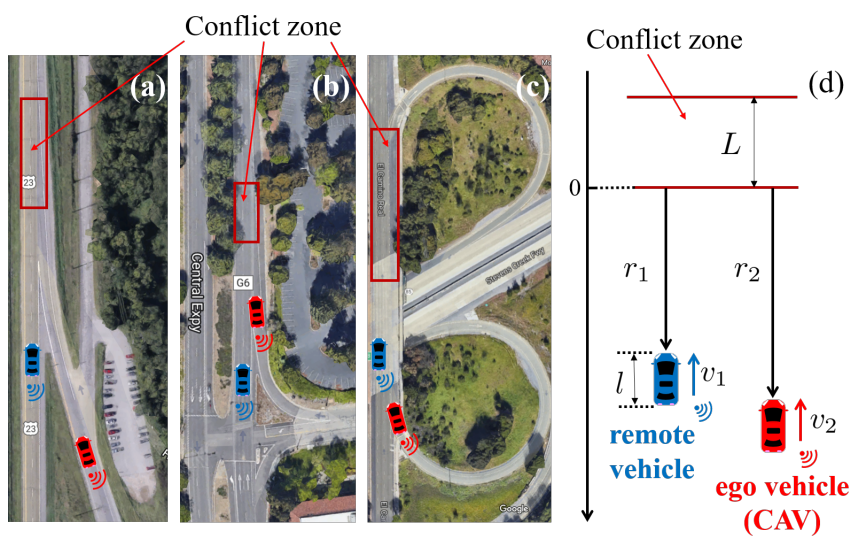

Fig. 2. Cooperative maneuvers at (a) highway on-ramp, (b) expressway entrance, (c) highway off-ramp. (d) Model used for conflict analysis. A conflict happens if both vehicles are present (even partially) in the conflict zone, and thus, the key parameter $s=L+l$ is the sum of conflict zone length and the vehicle length.

\section{Modeling Vehicle Dynamics}

Consider the scenarios in Fig. 2(a)-(c) where the conflict zones are indicated by red rectangles near the end of the ramps. The length of the conflict zone represents a safe distance between the vehicles which may vary according to the road configuration and traffic conditions. Here, to simplify the matter, we ignore the lateral dynamics of the vehicles and consider the model shown in Fig. 2(d). The distances of the remote vehicle and ego vehicle from the conflict zone are denoted by $r_{1}$ and $r_{2}$ while their longitudinal velocities are $v_{1}$ and $v_{2}$, respectively. Here, we use subscript 1 to refer to the remote vehicle and subscript 2 to refer to the ego vehicle. The length of the conflict zone is denoted by $L$, the length of both vehicles is $l$, and we define $s:=L+l$ to account for the length of the vehicles in case of conflict. Note that the conflict analysis presented below can be adapted to many other conflict scenarios with conflict zones of various sizes and shapes by appropriately selecting the conflict zone parameters.

To highlight the main idea of conflict analysis, we adopt a simple model for the longitudinal dynamics of the vehicles. By neglecting the air drag and the rolling resistance we have

$$
\begin{aligned}
& \dot{r}_{1}=-v_{1}, \\
& \dot{v}_{1}=\operatorname{sat}\left(u_{1}\right), \\
& \dot{r}_{2}=-v_{2}, \\
& \dot{v}_{2}=\operatorname{sat}\left(u_{2}\right) .
\end{aligned}
$$

Here the dot represents the derivatives with respect to time $t$ and the negative signs appear since the vehicles are traveling towards the conflict zone. Moreover, $u_{1}$ and $u_{2}$ represent the control inputs and the saturation function is included to take into account the acceleration limits of both the ego and remote vehicles.

Assuming that the velocity is between the assigned limits $v \in\left(v_{\min }, v_{\max }\right)$, we have

$$
\operatorname{sat}(u)= \begin{cases}a_{\min } & \text { if } u \in\left(-\infty, a_{\min }\right], \\ u & \text { if } u \in\left(a_{\min }, a_{\max }\right), \\ a_{\max } & \text { if } u \in\left[a_{\max }, \infty\right) .\end{cases}
$$

TABLE I

PARAMETERS VALUES USED IN THE PAPER.

\begin{tabular}{|c|c||c|c|}
\hline$L$ & $20[\mathrm{~m}]$ & $l$ & $5[\mathrm{~m}]$ \\
$a_{\min , 1}$ & $-4\left[\mathrm{~m} / \mathrm{s}^{2}\right]$ & $a_{\min , 2}$ & $-8\left[\mathrm{~m} / \mathrm{s}^{2}\right]$ \\
$a_{\max , 1}$ & $2\left[\mathrm{~m} / \mathrm{s}^{2}\right]$ & $a_{\max , 2}$ & $4\left[\mathrm{~m} / \mathrm{s}^{2}\right]$ \\
$v_{\min , 1}$ & $20[\mathrm{~m} / \mathrm{s}]$ & $v_{\min , 2}$ & $0[\mathrm{~m} / \mathrm{s}]$ \\
$v_{\max , 1}$ & $35[\mathrm{~m} / \mathrm{s}]$ & $v_{\max , 2}$ & $35[\mathrm{~m} / \mathrm{s}]$ \\
\hline
\end{tabular}

For $v=v_{\min }$, we substitute $a_{\min }$ with 0 , since the vehicle would not decelerate in this case. Similarly, when $v=v_{\max }$, we substitute $a_{\max }$ with 0 , since the vehicle would not exceed the speed limit. Indeed, the acceleration limits and the speed limits may be different for different vehicles; see Table I. Notice that $v_{\min , 2}$ is set to zero, that is, the ego vehicle is allowed to stop along the ramp. Since we are controlling the motion of the ego vehicle, we also assume that we are able to assign acceleration limits that correspond to emergency braking and full-throttle acceleration [4]. On the other hand, we do not have control over the remote vehicle (it may be a human-driven vehicle), and we assume that its acceleration and speed limits correspond to reasonable highway driving behavior. Note that this analysis could also be carried out for different parameter values.

Now we define the state vector

$$
x:=\left[\begin{array}{llll}
r_{1} & v_{1} & r_{2} & v_{2}
\end{array}\right]^{\top} \in \Omega,
$$

where $\Omega:=[-s, \infty) \times\left[v_{\min , 1}, v_{\max , 1}\right] \times[-s, \infty) \times\left[0, v_{\max , 2}\right]$. These states can be made available for both vehicles via V2X connectivity (e.g., a status sharing message from the remote vehicle shall contain $\left[\begin{array}{ll}r_{1} & v_{1}\end{array}\right]^{\top}$ ). However, when designing the decision making and control algorithms, we can only prescribe the input $u_{2}$ of the ego vehicle. The input $u_{1}$ of the remote vehicle cannot be prescribed, only its bounds (given by the saturation function) are assumed to be known. When the intent of the remote vehicle is shared via V2X communication, the ego vehicle may utilize this information to have a better prediction of the remote vehicle's motion, but the one still has no control over $u_{1}$. That is, the overall system (1) is not controllable, and our goal is to ensure that no conflict occurs under such assumptions.

\section{Conflict Analysis}

In this section, we provide a rigorous definition of conflict using mathematical logic and the model constructed above. Then, we calculate domains of different qualitative behaviors in the state space and display them on conflict charts.

As mentioned above, a conflict occurs if both vehicles appear in the conflict zone at the same time. This can be formalized as the proposition

$$
C:=\left\{\exists t, r_{1}(t) \in[-s, 0] \wedge r_{2}(t) \in[-s, 0]\right\},
$$

and a non-conflicting maneuver is given by

$$
\neg C=\left\{\forall t, r_{1}(t) \notin[-s, 0] \vee r_{2}(t) \notin[-s, 0]\right\},
$$

where we use the symbols $\wedge$ (and), $\vee$ (or), and $\neg$ (negation). This definition can be generalized for more than two vehicles and for different traffic scenarios such as intersections, roundabouts, and lane changes. 
We can decouple $\neg C$ into two cases where the ego vehicle merges ahead of the remote vehicle and where it merges behind:

$$
\begin{aligned}
& P:=\left\{\exists t, r_{1}(t)=0 \wedge r_{2}(t)<-s\right\}, \\
& Q:=\left\{\exists t, r_{1}(t)=-s \wedge r_{2}(t)>0\right\} .
\end{aligned}
$$

Proposition $P$ describes that by the time the remote vehicle enters the conflict zone, the ego vehicle has already passed it, while proposition $Q$ states that by the time the ego vehicle enters the conflict zone, the remote vehicle has already passed it. Furthermore, one can show that the relationship $P \vee Q \Longleftrightarrow \neg C$ holds (see Appendix A), leading to the definition of non-conflicting maneuver.

Definition 1. Given the dynamics (1), a maneuver is nonconflicting if proposition $P$ or proposition $Q$ is true.

Again, this definition may be extended to more than two vehicles by defining pairwise conflicts and can be generalized to a large variety of traffic scenarios.

Proposition $P$ can be decomposed into three cases:

(1) No-conflict with respect to $P$ : the ego vehicle is able to merge ahead without conflict independent of the subsequent motion of the remote vehicle.

(2) Uncertain with respect to $P$ : the ego vehicle may be able to merge ahead without conflict depending on the subsequent motion of the remote vehicle.

(3) Conflict with respect to $P$ : the ego vehicle is not able to merge ahead without conflict independent of the subsequent motion of the remote vehicle.

Mathematically these can be formulated as disjoint sets in state space:

$$
\begin{gathered}
\mathcal{P}_{\mathrm{g}}:=\left\{x(0) \in \Omega \mid \forall u_{1}(t), \exists u_{2}(t), P \text { for } t>0\right\}, \\
\mathcal{P}_{\mathrm{y}}:=\left\{x(0) \in \Omega \mid\left(\exists u_{1}(t), \forall u_{2}(t), \neg P \text { for } t>0\right) \wedge\right. \\
\left.\left(\exists u_{1}(t), \exists u_{2}(t), P \text { for } t>0\right)\right\}, \\
\mathcal{P}_{\mathrm{r}}:=\left\{x(0) \in \Omega \mid \forall u_{1}(t), \forall u_{2}(t), \neg P \text { for } t>0\right\},
\end{gathered}
$$

and we refer to these as no-conflict set, uncertain set, and conflict set with respect to merge ahead, respectively. The subscripts $\mathrm{g}, \mathrm{y}$ and $\mathrm{r}$ correspond to the colors green, yellow, and red that will be used to visualize the sets in state space. Similarly, based on proposition $Q$, the state space can be decomposed into no-conflict set, uncertain set, and conflict set with respect to the merge behind, that is,

$$
\begin{aligned}
& Q_{\mathrm{g}}:=\left\{x(0) \in \Omega \mid \forall u_{1}(t), \exists u_{2}(t), Q \text { for } t>0\right\}, \\
& Q_{\mathrm{y}}:=\left\{x(0) \in \Omega \mid\left(\exists u_{1}(t), \forall u_{2}(t), \neg Q \text { for } t>0\right) \wedge\right. \\
&\left.\left(\exists u_{1}(t), \exists u_{2}(t), Q \text { for } t>0\right)\right\}, \\
& \mathcal{Q}_{\mathrm{r}}:=\left\{x(0) \in \Omega \mid \forall u_{1}(t), \forall u_{2}(t), \neg Q \text { for } t>0\right\} .
\end{aligned}
$$

Note that the first and second predicates in (8) are the negation of the predicates in (7) and (9), that is,

$$
\begin{aligned}
\left(\exists u_{1}, \forall u_{2}, \neg P\right) & \Longleftrightarrow \neg\left(\forall u_{1}, \exists u_{2}, P\right), \\
\left(\exists u_{1}, \exists u_{2}, P\right) & \Longleftrightarrow \neg\left(\forall u_{1}, \forall u_{2}, \neg P\right),
\end{aligned}
$$

implying that $\mathcal{P}_{\mathrm{g}}, \mathcal{P}_{\mathrm{y}}$, and $\mathcal{P}_{\mathrm{r}}$ are pairwise disjoint and giving $\mathcal{P}_{\mathrm{g}} \cup \mathcal{P}_{\mathrm{y}} \cup \mathcal{P}_{\mathrm{r}}=\Omega$. Similar relationships also exist in (10-12).
We remark that the formal logic-based set description can be naturally extended to more complex conflict scenarios (e.g., more vehicles or lanes), which guarantees mathematical strictness while keeping the definition concise and scalable. We also emphasize that the decoupling of the propositions $P$ and $Q$ breaks down the problem of preventing conflict into the merge ahead scenario and the merge behind scenario.

\section{A. Conflict charts}

Using model (1), the boundaries between the domains $\mathcal{P}_{\mathrm{g}}$, $\mathcal{P}_{\mathrm{y}}$, and $\mathcal{P}_{\mathrm{r}}$ in state space can be calculated analytically, and the same holds for $Q_{\mathrm{g}}, Q_{\mathrm{y}}$, and $Q_{\mathrm{r}}$. By superimposing these domains, we can create conflict charts that separate the state space into no-conflict, uncertain, and conflict domains.

First, we focus on the sets $\mathcal{P}_{\mathrm{g}}, \mathcal{P}_{\mathrm{y}}$, and $\mathcal{P}_{\mathrm{r}}$. If $r_{1}(0) \in[-s, 0]$, the remote vehicle starts in the conflict zone and the ego vehicle has no chance to merge ahead without conflict. When $r_{1}(0) \in(0, \infty)$, we can describe two boundaries, $r_{2}=p_{1}\left(r_{1}, v_{1}, v_{2}\right)$ and $r_{2}=p_{2}\left(r_{1}, v_{1}, v_{2}\right)$, which separate $\mathcal{P}_{\mathrm{g}}, \mathcal{P}_{\mathrm{y}}$, and $\mathcal{P}_{\mathrm{r}}$ as visualized in the $\left(v_{2}, r_{2}\right)$-plane in Fig. 3(a). These boundaries are derived by considering that by the time the remote vehicle enters the conflict zone the ego vehicle just exits while applying the input bounds $\left(u_{1}(t), u_{2}(t)\right) \equiv\left(a_{\max , 1}, a_{\max , 2}\right)$ and $\left(u_{1}(t), u_{2}(t)\right) \equiv\left(a_{\min , 1}, a_{\max , 2}\right)$; see Appendix B.

It can be proven that $p_{2}\left(r_{1}, v_{1}, v_{2}\right) \geq p_{1}\left(r_{1}, v_{1}, v_{2}\right)$, $\forall r_{1} \in(0, \infty), v_{1} \in\left[v_{\min , 1}, v_{\max , 1}\right], v_{2} \in\left[0, v_{\max , 2}\right]$. Thus, the regions $\mathcal{P}_{\mathrm{g}}, \mathcal{P}_{\mathrm{y}}$, and $\mathcal{P}_{\mathrm{r}}$ are given by

$$
\begin{aligned}
& \mathcal{P}_{\mathrm{g}}=\left\{x \in \Omega \mid r_{2}<p_{1}\left(r_{1}, v_{1}, v_{2}\right)\right\}, \\
& \mathcal{P}_{\mathrm{y}}=\left\{x \in \Omega \mid p_{1}\left(r_{1}, v_{1}, v_{2}\right) \leq r_{2}<p_{2}\left(r_{1}, v_{1}, v_{2}\right)\right\}, \\
& \mathcal{P}_{\mathrm{r}}=\Omega \backslash\left(\mathcal{P}_{\mathrm{g}} \cup \mathcal{P}_{\mathrm{y}}\right),
\end{aligned}
$$

and these are shaded as green, yellow, and red in Fig. 3(a), respectively.

Similarly, consider the sets $Q_{g}, Q_{y}$, and $Q_{r}$. There are two boundaries related to proposition $Q: r_{2}=q_{1}\left(r_{1}, v_{1}, v_{2}\right)$ and $r_{2}=q_{2}\left(r_{1}, v_{1}, v_{2}\right)$; see Fig. 3(b). These are calculated by considering that by the time the remote vehicle exits the conflict zone, the ego vehicle just enters while applying the input bounds $\left(u_{1}(t), u_{2}(t)\right) \equiv\left(a_{\min , 1}, a_{\min , 2}\right)$ and $\left(u_{1}(t), u_{2}(t)\right) \equiv\left(a_{\max , 1}, a_{\min , 2}\right)$. Note that boundaries $q_{1}$ and $q_{2}$ overlap for $v_{2} \in\left[0,-t_{\mathrm{q} 2} a_{\min , 2}\right]$, where $t_{\mathrm{q} 2}$ represents the time needed for the remote vehicle to exit the conflict zone, with $u_{1}(t) \equiv a_{\max , 1}$; see (57-59) in Appendix B. One can then prove that, $q_{1}\left(r_{1}, v_{1}, v_{2}\right) \geq q_{2}\left(r_{1}, v_{1}, v_{2}\right), \quad \forall r_{1} \in[-s, \infty)$, $v_{1} \in\left[v_{\min , 1}, v_{\max , 1}\right], v_{2} \in\left(-t_{\mathrm{q} 2} a_{\min , 2}, \infty\right)$. Thus, the regions $Q_{\mathrm{g}}, Q_{\mathrm{y}}$, and $Q_{\mathrm{r}}$ are

$$
\begin{aligned}
& \mathcal{Q}_{\mathrm{g}}=\left\{x \in \Omega \mid r_{2}>q_{1}\left(r_{1}, v_{1}, v_{2}\right)\right\} \\
& \mathcal{Q}_{\mathrm{y}}=\left\{x \in \Omega \mid q_{2}\left(r_{1}, v_{1}, v_{2}\right)<r_{2} \leq q_{1}\left(r_{1}, v_{1}, v_{2}\right)\right\}, \\
& \mathcal{Q}_{\mathrm{r}}=\Omega \backslash\left(\mathcal{Q}_{\mathrm{g}} \cup \mathcal{Q}_{\mathrm{y}}\right) .
\end{aligned}
$$

These regions are shaded as green, yellow, and red in Fig. 3(b), respectively.

Figure 3(d)-(e) visualize the corresponding boundaries and regions in the $\left(r_{1}, r_{2}\right)$-plane, where the red square at the bottom left corner represents the conflict zone. Note that 

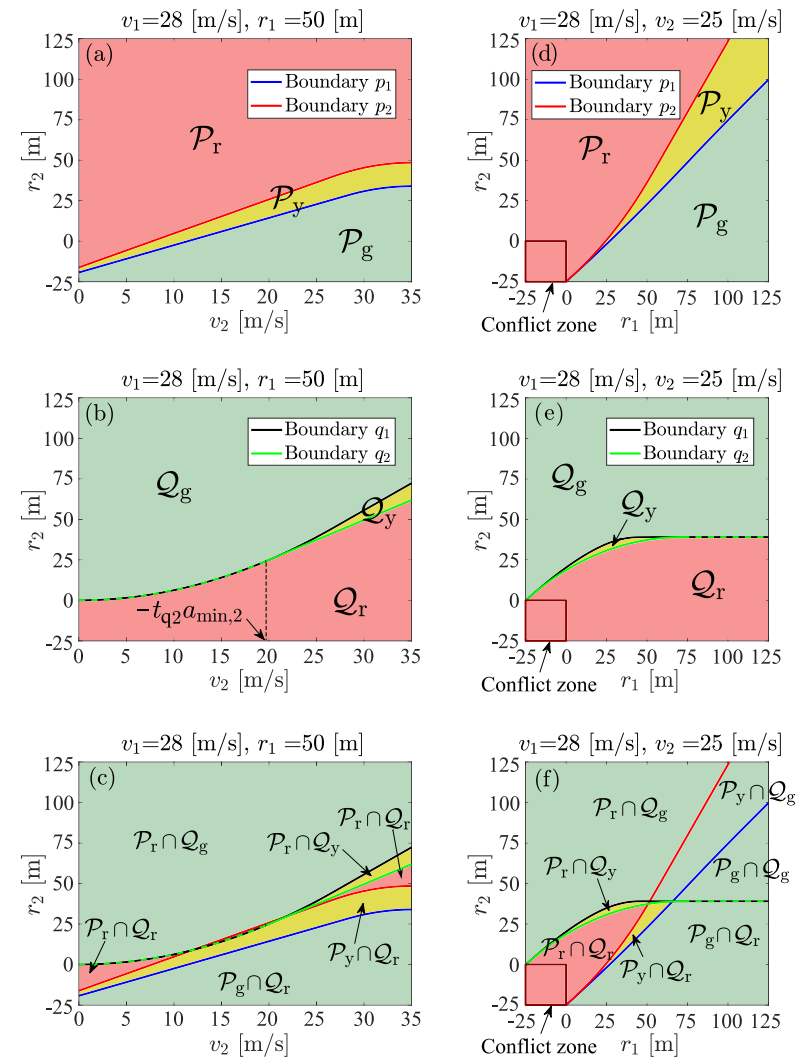

Fig. 3. (a)-(c) Conflict charts in the $\left(v_{2}, r_{2}\right)$-plane for given $v_{1}, r_{1}$ values as indicated. (a) Conflict charts for merge ahead. (b) Conflict charts for merge behind. (c) Unified conflict charts combining (a) and (b). (d)-(f) Conflict charts in the $\left(r_{1}, r_{2}\right)$-plane for given $v_{1}, v_{2}$ values as indicated.

the sets partitioning the state space are obtained analytically using the simple model (1) without resistance terms. With more complicated models, analytical calculations may not be possible and numerical tools may be needed. Developing such tools, however, is beyond the scope of this paper.

Having introduced the boundaries and regions related to $P$ and $Q$ separately, we combine them together in Fig. 3(c) and (f). Each region in these graphs is given by the intersection of a set related to $P$ and a set related to $Q$. We color the regions as follows: combining a green region with any other region gives green; combining a yellow region with a yellow or red region gives yellow; combining two red regions gives red. We refer to these as unified conflict charts. Such conflict charts can be built by the ego vehicle once a status of the remote vehicle is received (via V2X) since the positions and velocities of both vehicles are required to determine the boundaries $p_{1}$, $p_{2}, q_{1}, q_{2}$ partitioning the state space.

In the unified conflict chart in Fig. 3(f), the red-shaded region corresponds to the "capture set" computed in [15], [17]. However, here the decision making boundaries further divide the rest of the state space into different regions, enabling the ego vehicle to make decisions (and act on them) long before approaching the capture set. As will be explained below, the decision in the green region below the blue boundary shall be "merge ahead" and in the green region above the blue boundary shall be "merge behind" in order to prevent conflict independent of the future behavior of the remote vehicle.
Fig. 4(a)-(d), show a sequence of charts in the $\left(v_{2}, r_{2}\right)$ plane for fixed $v_{1}$ and different $r_{1}$ values as indicated, which illustrates the evolution of the boundaries while the remote vehicle approaches the conflict zone with constant speed. On the other hand, Fig. 4(e)-(h) show a sequence of conflict charts in the $\left(r_{1}, r_{2}\right)$-plane when the remote vehicle travels at constant speed while the ego vehicle is accelerating. Indeed, the sequences of two-dimensional conflict charts depend on the behaviors of the ego and remote vehicles.

\section{B. Communication range and decision making rule}

The conflict charts introduced above provide a general tool for decision making. These, however, rely on having motion information available from the remote vehicle, which, in most cases, cannot be obtained using optical sensors, but require the use of V2X communication. Here we provide conditions for the required range of $\mathrm{V} 2 \mathrm{X}$ communication in order to guarantee conflict free maneuvers.

For initial conditions $x(0) \in \mathcal{P}_{\mathrm{g}} \cup \mathrm{Q}_{\mathrm{g}}$ in the green region, there exists a controller $u_{2}(t)$ such that conflict can be prevented for $t>0$, that is, $x(t) \in \mathcal{P}_{\mathrm{g}} \cup Q_{\mathrm{g}}$. The following theorem relates this to a communication range requirement.

Theorem 1. The statement $x(0) \in \mathcal{P}_{\mathrm{g}} \cup \mathcal{Q}_{\mathrm{g}}$ holds for $r_{1}(0) \geq r_{1}^{*}$ where

$$
r_{1}^{*}=\max \left\{\underline{r}_{1}, \bar{r}_{1}\right\},
$$

and

$$
\begin{gathered}
\underline{r}_{1}= \begin{cases}\sqrt{\frac{2 s}{a_{\max , 2}}} v_{\max , 1}, & \text { if } \quad s a_{\max , 2} \leq \frac{1}{2} v_{\max , 2}^{2}, \\
\left(s+\frac{v_{\max , 2}^{2}}{2 a_{\max , 2}}\right) \frac{v_{\max , 1}}{v_{\max , 2}}, & \text { otherwise },\end{cases} \\
\bar{r}_{1}=\left(s-\frac{v_{\max , 2}^{2}}{2 a_{\min , 2}}\right) \frac{v_{\max , 1}}{v_{\max , 2}}
\end{gathered}
$$

Proof. See Appendix C.

The proof is based on showing that, if $r_{1}(0) \geq r_{1}^{*}$, then $p_{1}\left(r_{1}, v_{1}, v_{2}\right) \geq q_{1}\left(r_{1}, v_{1}, v_{2}\right)$ independent of the values of $v_{1}$ and $v_{2}$, meaning that the system state is guaranteed to stay in the green no-conflict domain $\mathcal{P}_{\mathrm{g}} \cup \mathcal{Q}_{\mathrm{g}}$. The theorem implies that if the ego vehicle receives a status packet from the remote vehicle (at $t=0$ ) when the latter is at least $r_{1}^{*}$ distance away from the conflict zone, then independent of the input $u_{1}(t)$, $t \geq 0$, there exists a controller $u_{2}(t), t \geq 0$ which can prevent conflicts for $t>0$. For the parameters in Table I, we have $r_{1}^{*}=124[\mathrm{~m}]$ which is possible to satisfy with current V2X technologies. Fig. 5(a)-(c) show how the communication range $r_{1}^{*}$ is affected by the parameters of the ego vehicle. Notice that with lower capability of accelerating and decelerating, larger communication range is required to prevent conflict. This also implies that having a larger communication range may help one to improve passenger comfort.

Now we propose a decision making rule for the ego vehicle:

$$
\text { decision }= \begin{cases}\text { merge ahead, } & \text { if } x(0) \in \mathcal{P}_{\mathrm{g}}, \\ \text { merge behind, } & \text { if } x(0) \notin \mathcal{P}_{\mathrm{g}} \wedge x(0) \in \mathcal{Q}_{\mathrm{g}} .\end{cases}
$$



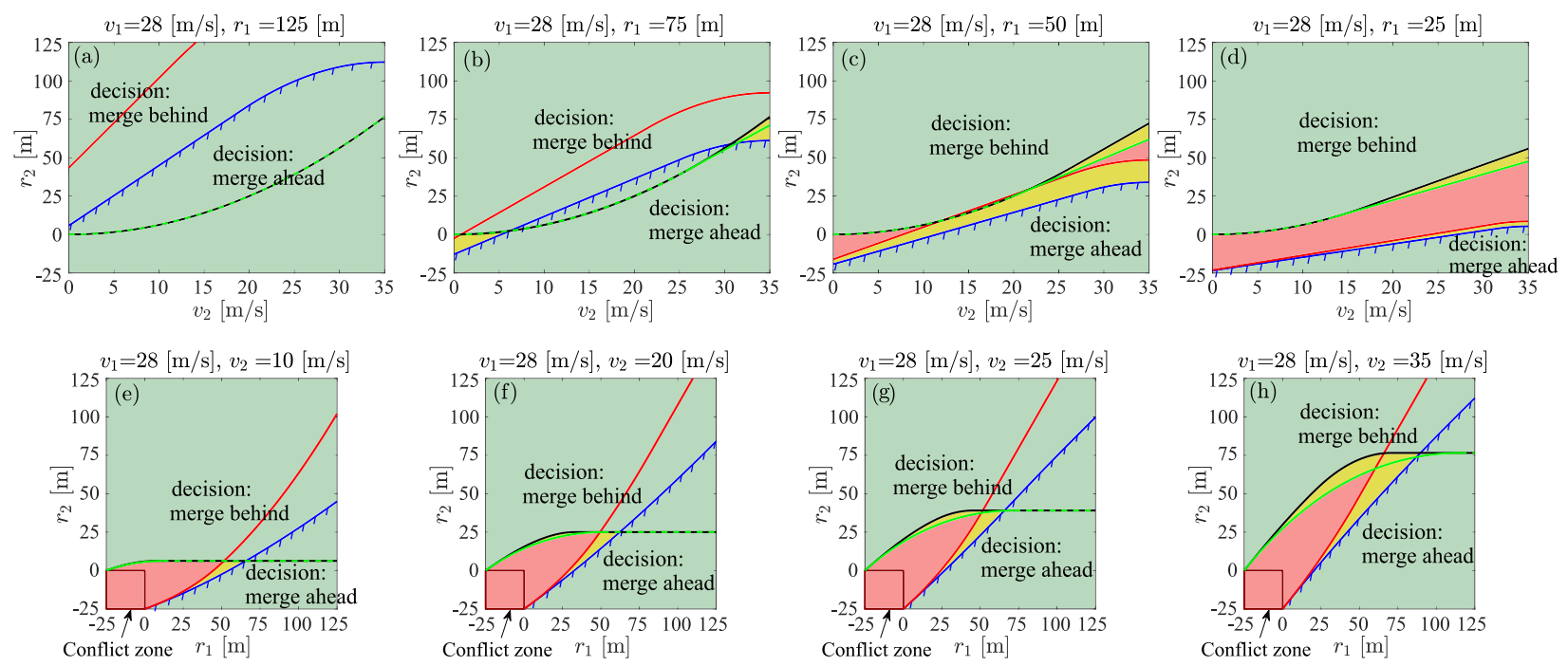

Fig. 4. (a)-(d) Conflict charts in the $\left(v_{2}, r_{2}\right)$-plane when the remote vehicle is approaching with a constant speed. (e)-(h) Conflict charts in the $\left(r_{1}, r_{2}\right)$-plane when the remote vehicle travels at constant speed while the ego vehicle is accelerating. According to the decision making rule (23), the decision in the green region below the blue boundary is merge ahead while the decision in the green region above the blue boundary is merge behind.
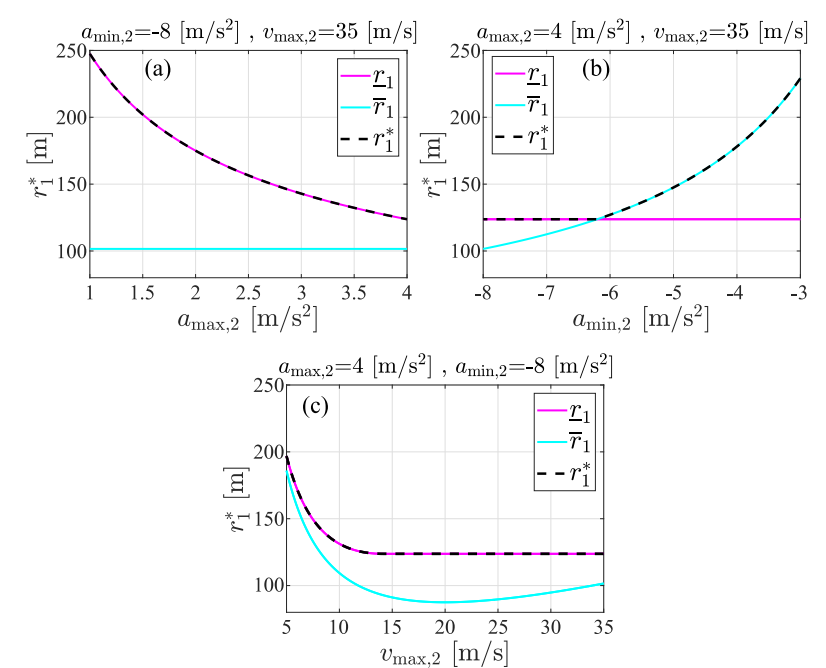

Fig. 5. Communication range as a function of (a) $a_{\max , 2}$, (b) $a_{\min , 2}$ and (c) $v_{\max , 2}$, where the parameters of the remote vehicle are given in Table I.

That is, if the initial state $x(0) \in \mathcal{P}_{\mathrm{g}}$, then independent of the motion of the remote vehicle, the ego vehicle is able to merge ahead without conflict, and the decision maximizes its time efficiency. On the other hand, if $x(0) \notin \mathcal{P}_{\mathrm{g}}$ and $x(0) \in \mathcal{Q}_{\mathrm{g}}$, then independent of the motion of the remote vehicle, the ego vehicle is able to merge behind without conflict.

If the communication range requirement in Theorem 1 is satisfied, a non-conflicting decision is guaranteed by (23) for any initial state of the ego vehicle. Also, (23) suggests that for $x(0) \in \mathcal{P}_{\mathrm{g}} \cap Q_{\mathrm{g}}$, the ego vehicle still chooses to merge ahead to increase its time efficiency. If the communication range requirement is not satisfied (see, e.g., Fig. 4(b)-(d)), the decision making rule (23) can still be applied. However, if the initial state $x(0) \notin \mathcal{P}_{\mathrm{g}} \cup \mathcal{Q}_{\mathrm{g}}$ then a definite decision cannot be made due to the unknown future behaviors of the remote vehicle. In the next section, we provide a potential solution to this problem by making the intent of the remote vehicle available via V2X connectivity.

\section{CONFLICT ANALYSiS With INTENT INFORMATION}

In this section, we show that obtaining intent information from the remote vehicle can help the ego vehicle to make decisions since it decreases the uncertainty regarding the future behavior of the remote vehicle. We also demonstrate that making better decisions may significantly improve the time efficiency of the ego vehicle when merging. We note again that intent sharing is beneficial to cooperative driving when it is shared by a vehicle with at least partial automation; see Fig. 1.

First, we define the intent of the remote vehicle under the scenario described in Section II.

Definition 2. Given the dynamics $(1,2)$, the intent of the remote vehicle is a restricted velocity domain $v_{1}(t) \in\left[\underline{v}_{1}, \bar{v}_{1}\right]$ and acceleration (input) domain $u_{1}(t) \in\left[\underline{a}_{1}, \bar{a}_{1}\right]$ over some time period $t \in[0, \Delta t]$, where $v_{\min , 1} \leq \underline{v}_{1} \leq \bar{v}_{1} \leq v_{\max , 1}$ and $a_{\min , 1} \leq \underline{a}_{1} \leq \bar{a}_{1} \leq a_{\max , 1}$.

For example, in a highway driving scenario with cruise control, an intent message may encode that for the next $\Delta t=15$ seconds, the remote vehicle will travel with a speed between $\underline{v}_{1}=27[\mathrm{~m} / \mathrm{s}]$ and $\bar{v}_{1}=29[\mathrm{~m} / \mathrm{s}]$ while limiting its acceleration between $\underline{a}_{1}=-1\left[\mathrm{~m} / \mathrm{s}^{2}\right]$ and $\bar{a}_{1}=1\left[\mathrm{~m} / \mathrm{s}^{2}\right]$.

For simplicity, we assume that the time interval $\Delta t$ of the intent covers the time horizon until the remote vehicle clears the conflict zone. This assumption is realistic for merge, intersection crossing, and lane change maneuvers as those typically last for a few seconds. Thus, when the intent information is available, the boundaries in the conflict charts can be re-calculated using the formulas in Appendix B with the remote vehicle's velocity and acceleration limits given by its intent. Let us use $r_{2}=\bar{p}_{1}\left(r_{1}, v_{1}, v_{2}\right), r_{2}=\bar{p}_{2}\left(r_{1}, v_{1}, v_{2}\right)$, $r_{2}=\bar{q}_{1}\left(r_{1}, v_{1}, v_{2}\right)$, and $r_{2}=\bar{q}_{2}\left(r_{1}, v_{1}, v_{2}\right)$ to denote the new boundaries. One can then define no-conflict, uncertain, and conflict sets with intent information. For proposition $P$ we 

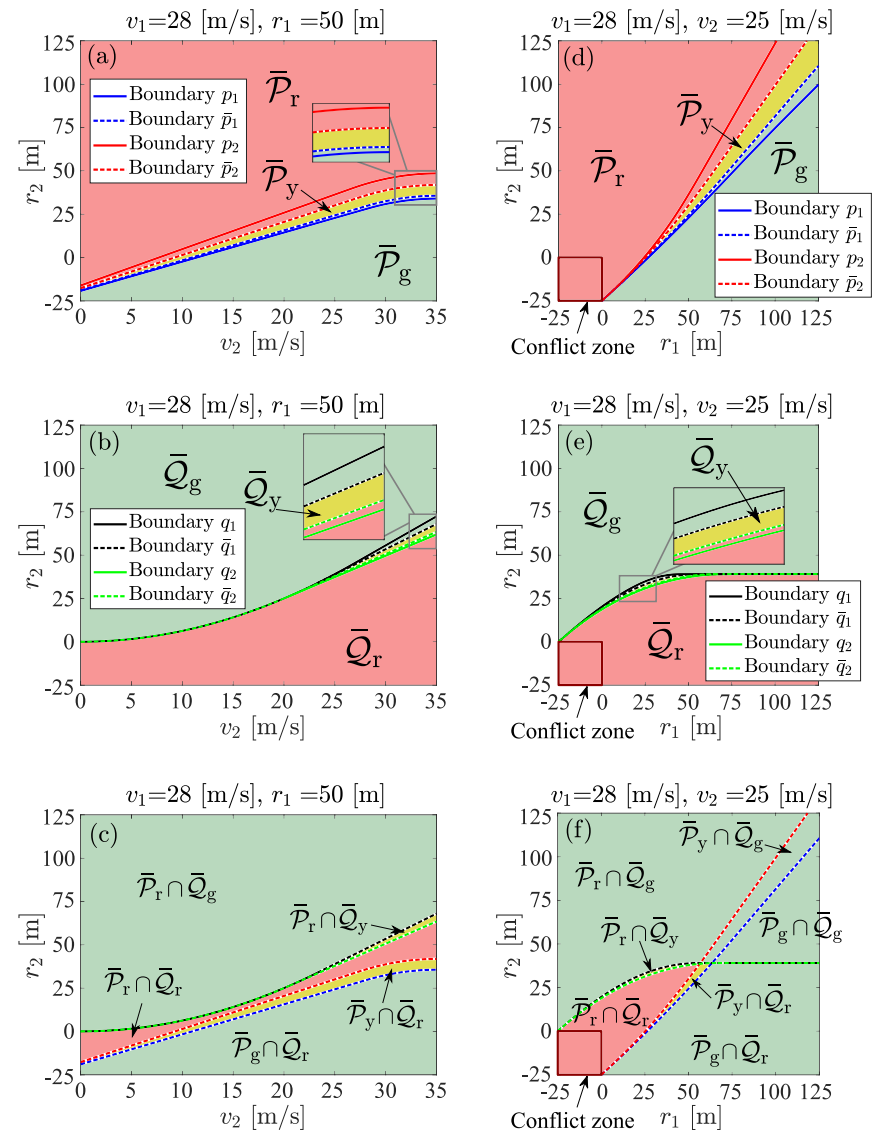

Fig. 6. (a)-(c) Conflict charts in the $\left(v_{2}, r_{2}\right)$-plane given the intent of the remote vehicle: $v_{1} \in[25,30][\mathrm{m} / \mathrm{s}]$ and $u_{1} \in[-2,1]\left[\mathrm{m} / \mathrm{s}^{2}\right]$. (a) Conflict charts for merge ahead. (b) Conflict charts for merge behind. (c) Unified conflict charts combining (a) and (b). (d)-(f) Conflict charts in the $\left(r_{1}, r_{2}\right)$ plane given the same intent of the remote vehicle.

have

$$
\begin{aligned}
& \overline{\mathcal{P}}_{\mathrm{g}}=\left\{x \in \Omega \mid r_{2}<\bar{p}_{1}\left(r_{1}, v_{1}, v_{2}\right)\right\}, \\
& \overline{\mathcal{P}}_{\mathrm{y}}=\left\{x \in \Omega \mid \bar{p}_{1}\left(r_{1}, v_{1}, v_{2}\right) \leq r_{2}<\bar{p}_{2}\left(r_{1}, v_{1}, v_{2}\right)\right\}, \\
& \overline{\mathcal{P}}_{\mathrm{r}}=\Omega \backslash\left(\overline{\mathcal{P}}_{\mathrm{g}} \cup \overline{\mathcal{P}}_{\mathrm{y}}\right),
\end{aligned}
$$

cf. (14-16), while for proposition $Q$ we obtain

$$
\begin{aligned}
& \overline{\mathrm{Q}}_{\mathrm{g}}=\left\{x \in \Omega \mid r_{2}>\bar{q}_{1}\left(r_{1}, v_{1}, v_{2}\right)\right\} \\
& \overline{\mathrm{Q}}_{\mathrm{y}}=\left\{x \in \Omega \mid \bar{q}_{2}\left(r_{1}, v_{1}, v_{2}\right)<r_{2} \leq \bar{q}_{1}\left(r_{1}, v_{1}, v_{2}\right)\right\}, \\
& \overline{\mathrm{Q}}_{\mathrm{r}}=\Omega \backslash\left(\overline{\mathrm{Q}}_{\mathrm{g}} \cup \overline{\mathrm{Q}}_{\mathrm{y}}\right),
\end{aligned}
$$

cf. (17)-(19).

The following Theorem gives the relationships between the sets with and without intent information.

Theorem 2. Given the intent of the remote vehicle, we obtain

$$
\begin{aligned}
& \mathcal{P}_{\mathrm{g}} \subseteq \overline{\mathcal{P}}_{\mathrm{g}}, \mathcal{P}_{\mathrm{y}} \supseteq \overline{\mathcal{P}}_{\mathrm{y}}, \text { and } \mathcal{P}_{\mathrm{r}} \subseteq \overline{\mathcal{P}}_{\mathrm{r}}, \\
& \mathcal{Q}_{\mathrm{g}} \subseteq \overline{\mathcal{Q}}_{\mathrm{g}}, \mathcal{Q}_{\mathrm{y}} \supseteq \overline{\mathcal{Q}}_{\mathrm{y}}, \text { and } \mathrm{Q}_{\mathrm{r}} \subseteq \overline{\mathcal{Q}}_{\mathrm{r}} .
\end{aligned}
$$

\section{Proof. See Appendix D.}

The relationships (30) and (31) reveal that with the intent both the no-conflict and conflict sets (with respect to $P$ and $Q$ ) expand, while the uncertain sets (with respect to $P$ and $Q$ ) shrink. These are illustrated in the conflict charts in Fig. 6(a) and (d), and Fig. 6(b) and (e), respectively.
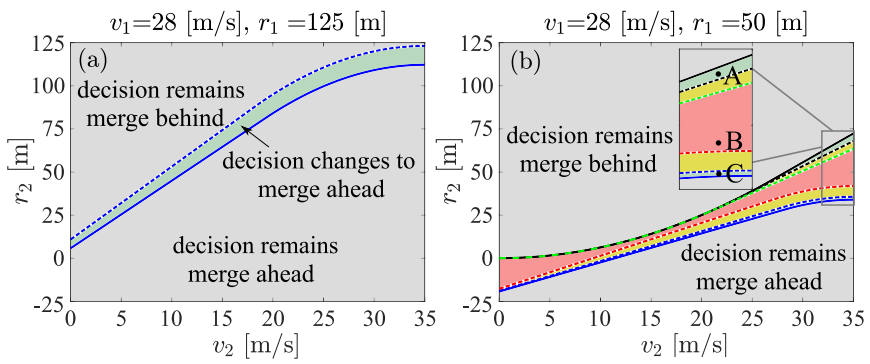

Fig. 7. Change of decision charts under the same intent as in Fig. 6 when the communication range requirement in Theorem 1 is satisfied (a) and when it is not satisfied (b). Conflict cannot be prevented without intent for the points marked A, B, and C. With intent the decisions become: A - merge behind; C - merge ahead; B - conflict is not preventable.

The following Corollary states that the uncertain sets $\overline{\mathcal{P}}_{\mathrm{y}}$ and $\bar{Q}_{\text {y }}$ disappear when considering a deterministic future motion (i.e., constant acceleration) for the remote vehicle.

Corollary 1. $\overline{\mathcal{P}}_{\mathrm{y}}=\bar{Q}_{\mathrm{y}}=\emptyset$ if the intent of the remote vehicle satisfies

$$
\left(\bar{a}_{1}=\underline{a}_{1}\right) \vee\left(\bar{v}_{1}=\underline{v}_{1}=v_{1}(t)\right) .
$$

This corollary can be proved by noticing that (32) leads to the relationships $\bar{p}_{1}\left(r_{1}, v_{1}, v_{2}\right)=\bar{p}_{2}\left(r_{1}, v_{1}, v_{2}\right)$ and $\bar{q}_{1}\left(r_{1}, v_{1}, v_{2}\right)=\bar{q}_{2}\left(r_{1}, v_{1}, v_{2}\right)$ between the boundaries.

Combining the conflict charts associated with propositions $P$ and $Q$, yields the following property for the unified conflict charts.

Corollary 2. Given the intent of the remote vehicle, in the unified conflict charts we have

$$
\begin{aligned}
& \mathcal{P}_{\mathrm{g}} \cup Q_{\mathrm{g}} \subseteq \overline{\mathcal{P}}_{\mathrm{g}} \cup \overline{\mathrm{Q}}_{\mathrm{g}}, \\
& \mathcal{P}_{\mathrm{r}} \cap Q_{\mathrm{r}} \subseteq \overline{\mathcal{P}}_{\mathrm{r}} \cap \overline{\mathcal{Q}}_{\mathrm{r}} .
\end{aligned}
$$

This corollary can be proved from (30) and (31) with basic set operations. Practically, (33) and (34) mean that with intent the green and red regions expand, resulting in smaller yellow region in the state space $\Omega$; cf. Fig. 6(c,f) with Fig. 3(c,f). That is, having intent information reduces the uncertainty in the decision making of the ego vehicle and provides additional danger awareness. Also, the decision making rule (23) can be adapted by using $\overline{\mathcal{P}}_{\mathrm{g}}$ and $\overline{\mathcal{Q}}_{\mathrm{g}}$ instead of $\mathcal{P}_{\mathrm{g}}$ and $\mathrm{Q}_{\mathrm{g}}$, respectively.

The charts in Fig. 7 quantify the benefits of using the intent information. These are obtained by superimposing the conflict charts with and without intent information; see Figs. 3, 4 and 6 . In the gray-shaded regions, the decision remains unchanged. Fig. 7(a) shows that when the communication range requirement $(20,21,22)$ is satisfied, there is a region where decision changes from merge behind to merge ahead. Fig. 7(b) depicts that when the communication range requirement is not satisfied, a previously yellow region changes to green and red. For example, points $\mathrm{A}, \mathrm{B}$, and $\mathrm{C}$ were in the yellow region in Fig. 3(c), while in Fig. 7(b) $\mathrm{A}$ and $\mathrm{C}$ are in the green region with decisions to merge behind and merge ahead, respectively. Point B is in the red region, giving the ego vehicle an awareness of danger that a conflict is not preventable. 


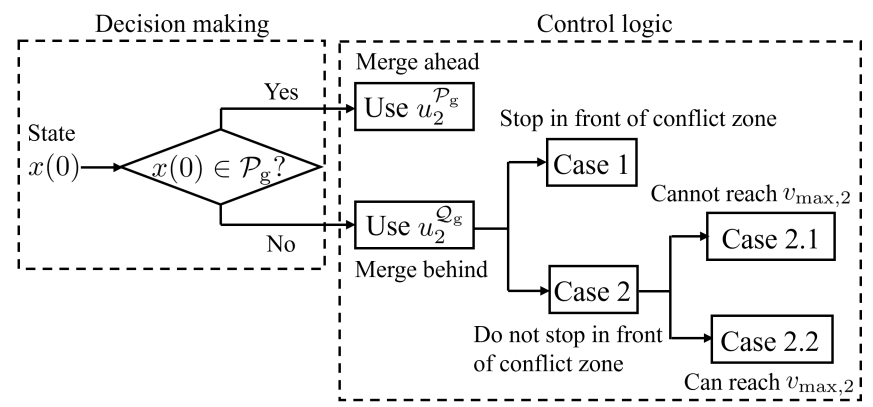

Fig. 8. Block diagram of the decision making and control logic of the CAV under the communication requirement in Theorem 1.
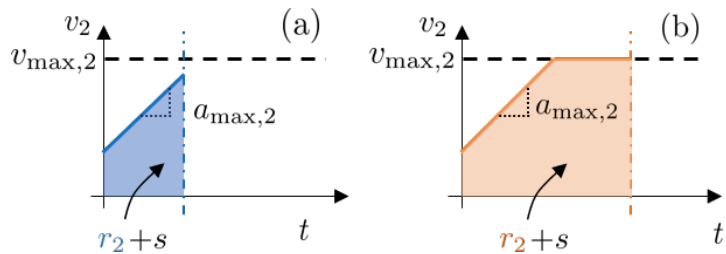

Fig. 9. Velocity profiles when applying controller $u_{2}^{\mathcal{P}_{\mathrm{g}}}$ when the speed limit $v_{\max , 2}$ is not reached (a) and when it is reached (b).

\section{Controller Design}

Based on the conflict analysis presented above, we design a controller for the ego vehicle to execute a conflict-free merge. Recall that this vehicle is considered to be automated and we refer to this as a connected automated vehicle (CAV) in this section. On the other hand, no automation is assumed for the remote vehicle, i.e., it can be human-driven or automated. Finally, we assume that the communication range requirement given in Theorem 1 holds, i.e., the CAV receives a status update from the remote vehicle before the latter one reaches the distance $r_{1}^{*}$ from the conflict zone.

To ensure a non-conflicting maneuver independent of the remote vehicle's future action $u_{1}(t)$, we propose the controller

$$
u_{2}(t)= \begin{cases}u_{2}^{\mathcal{P}_{\mathrm{g}}}, & \text { if decision }=\text { merge ahead } \\ u_{2}^{\mathcal{Q}_{\mathrm{g}}}, & \text { if decision }=\text { merge behind }\end{cases}
$$

for $t \geq 0$; see (23). Here, $u_{2}^{\mathcal{P}_{\mathrm{g}}}$ ensures that the ego vehicle merges ahead of the remote vehicle without conflict, and $u_{2}^{\mathcal{Q}_{\mathrm{g}}}$ ensures that it merges behind without conflict. A block diagram summarizing the decision making and control logic for the CAV is shown in Fig. 8, where the design of $u_{2}^{Q_{\mathrm{g}}}$ is divided into several cases as discussed below.

For merge ahead, the CAV chooses constant control input

$$
u_{2}^{\mathcal{P}_{\mathrm{g}}}=a_{\max , 2},
$$

since it makes the maneuver the most time-efficient (from the CAV's perspective). Note that according to the saturation function (2) in (1), the CAV's acceleration becomes zero once its velocity reaches $v_{\max , 2}$. Fig. 9 shows two different velocity profiles when $u_{2}^{\mathcal{P}_{\mathrm{g}}}$ is applied to pass the conflict zone. Panels (a) and (b) correspond to cases when $v_{\max , 2}$ is not reached and when it is reached, respectively.

For merge behind, the CAV uses the constant control input $u_{2}^{Q_{g}}$ that is calculated by assuming the worst-case scenario

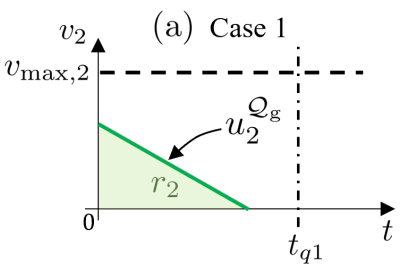

(b) Case 2.1
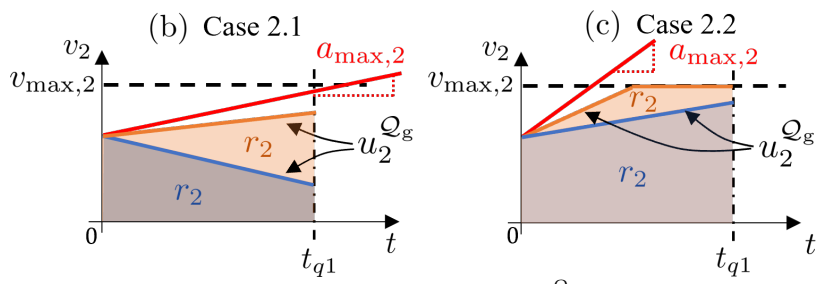

Fig. 10. Velocity profiles for the controller $u_{2}^{Q_{g}}$. The different panels correspond to the cases in Fig. 8.

$u_{1}(t)=a_{\min , 1}$ (or $u_{1}(t)=\underline{a}_{1}$ when intent is available). To maximize the time efficiency (of the CAV), we set $u_{2}^{Q_{\mathrm{g}}}$ such that the CAV arrives at the front edge of conflict zone at time $t_{\mathrm{q} 1}$, when the remote vehicle clears the conflict zone. In the formulas below, we drop the argument (0) when referring to the initial values of the state $x$, that is, we use $r_{1}, v_{1}, r_{2}, v_{2}$ instead of $r_{1}(0), v_{1}(0), r_{2}(0), v_{2}(0)$.

We distinguish two cases:

Case 1: $r_{2} \leq \frac{1}{2} t_{\mathrm{q} 1} v_{2} \Longrightarrow$ the $\mathrm{CAV}$ must stop at the front edge of the conflict zone. Then the control input is given by

$$
u_{2}^{Q_{g}}=-\frac{v_{2}^{2}}{2 r_{2}}
$$

which makes the CAV to stop right in front of the conflict zone at time $t_{\mathrm{q} 1}$; see Fig. 10(a) where the area below the curve is the distance $r_{2}$. Note that it can be shown from (37) that $u_{2}^{Q_{\mathrm{g}}}>a_{\min , 2}$.

Case 2: $r_{2}>\frac{1}{2} t_{\mathrm{q} 1} v_{2} \Longrightarrow$ the CAV does not need to stop at the front edge of conflict zone. For this case there are two subcases.

Case 2.1: $a_{\max , 2}<\left(v_{\max , 2}-v_{2}\right) / t_{\mathrm{q} 1} \Longrightarrow$ the CAV's speed cannot reach $v_{\max , 2}$ by time $t_{\mathrm{q} 1}$, not even by applying the maximum acceleration $a_{\max , 2}$; see Fig. 10(b). In this case we use

$$
\begin{aligned}
& u_{2}^{\mathrm{Q}_{\mathrm{g}}}= \\
& \begin{cases}\frac{2\left(r_{2}-v_{2} t_{\mathrm{q} 1}\right)}{t_{\mathrm{q} 1}^{2}}, & \text { if } r_{2} \in\left(\frac{1}{2} t_{\mathrm{q} 1} v_{2}, \frac{1}{2} t_{\mathrm{q} 1}^{2} a_{\max , 2}+v_{2} t_{\mathrm{q} 1}\right], \\
a_{\max , 2}, & \text { otherwise. }\end{cases}
\end{aligned}
$$

Case 2.2: $a_{\max , 2} \geq\left(v_{\max , 2}-v_{2}\right) / t_{\mathrm{q} 1} \Longrightarrow$ the CAV's speed can reach $v_{\max , 2}$ by time $t_{\mathrm{q} 1}$; see Fig. 10(c). In this case we use

$$
\begin{aligned}
& u_{2}^{\mathrm{Q}_{\mathrm{g}}}= \\
& \left\{\begin{array}{cl}
\frac{2\left(r_{2}-v_{2} t_{\mathrm{q} 1}\right)}{t_{\mathrm{q} 1}^{2}}, & \text { if } r_{2} \in\left(\frac{1}{2} t_{\mathrm{q} 1} v_{2}, \frac{1}{2} t_{\mathrm{q} 1}\left(v_{2}+v_{\max , 2}\right)\right] \\
\frac{\left(v_{\max , 2}-v_{2}\right)^{2}}{2\left(t_{\mathrm{q} 1} v_{\max , 2}-r_{2}\right)}, & \text { if } r_{2} \in\left(\frac{1}{2} t_{\mathrm{q} 1}\left(v_{2}+v_{\max , 2}\right),\right. \\
\quad & \left.\quad-\frac{\left(v_{\max , 2}-v_{2}\right)^{2}}{2 a_{\max , 2}}+t_{\mathrm{q} 1} v_{\max , 2}\right], \\
a_{\max , 2}, & \text { otherwise. }
\end{array}\right.
\end{aligned}
$$

Note that in Case 2.2 the control input $u_{2}^{Q_{g}}$ saturates once the CAV's speed reaches $v_{\max , 2}$. 
The proposed controller guarantees that region $\mathcal{P}_{\mathrm{g}}$ is invariant under $u_{2}^{\mathcal{P}_{\mathrm{g}}}$, and $\mathcal{Q}_{\mathrm{g}}$ is invariant under $u_{2}^{\mathcal{Q}_{\mathrm{g}}}$. That is, $\mathcal{P}_{\mathrm{g}} \cup Q_{\mathrm{g}}$ is control invariant under (35). Recall that $u_{2}^{Q_{\mathrm{g}}}$ is derived by using a status packet received from the remote vehicle at $t=0$ and it is assumed that the remote vehicle is applying $u_{1}(t) \equiv a_{\min , 1}\left(\right.$ or $\left.\underline{a}_{1}\right)$ along $t>0$. However, if the CAV receives status updates later, it can re-calculate (37), (38), and (39) using the most recent information, which results in larger value of $u_{2}^{Q_{\mathrm{g}}}$, and consequently, better time-efficiency for the CAV. The benefits of receiving frequent status updates will be shown in the next section.

\section{EXPERIMENTS AND SiMULATIONS}

In this section, we show two applications of the conflict analysis framework developed above. First, we present experimental results obtained in a closed test track to demonstrate that (i) the theoretical conflict chart matches with data collected using real vehicles, and (ii) our conflict analysis can help a CAV to prevent conflicts that a human driver could not avoid. Second, we present numerical simulations by using real highway data for the remote vehicle to demonstrate that a CAV is able to prevent conflict when using the proposed decision making and control algorithms. We also demonstrate that the performance of CAV can be significantly enhanced when it utilizes intent information of the remote vehicle.

\section{A. Experiments on test track with real vehicles}

The experiments were performed on the test track of the University of Michigan called Mcity with two real vehicles; see the aerial view in Fig. 11(a). The remote vehicle (blue) traveled along the main road while the ego vehicle (white) merged onto the main road within the yellow rectangle of 50 meters length. The conflict zone is indicated by the red

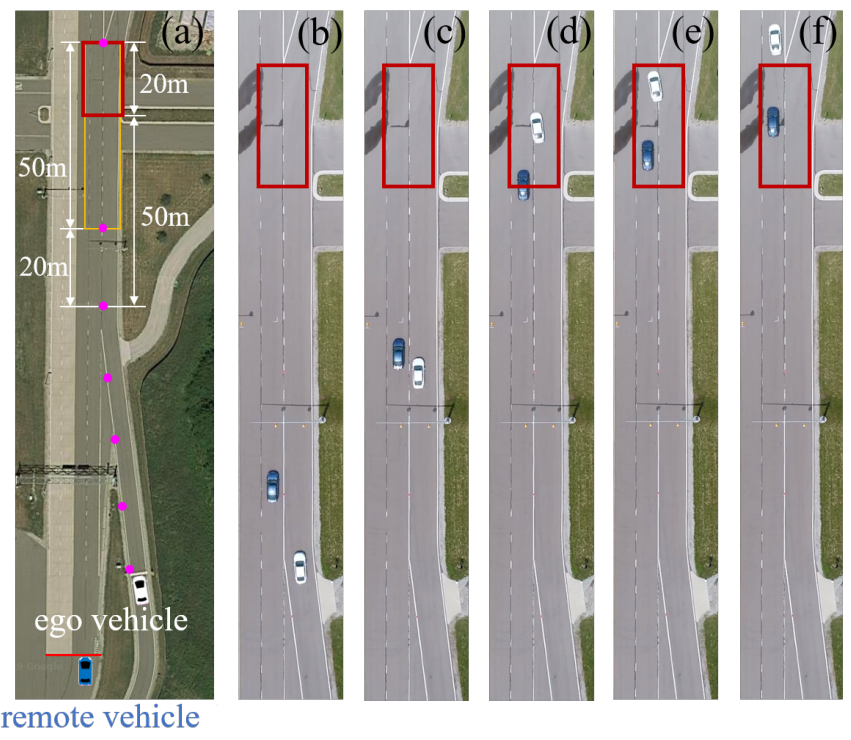

Fig. 11. Experimental setup. (a) Layout of the test track with merge zone (yellow rectangle), conflict zone (red rectangle), and start positions of the ego vehicle (magenta dots). (b-f) Snapshots from an experiment with panels (d) and (e) highlighting the conflict. rectangle of 20 meters length. Fig. 11(b)-(f) show snapshots from one of the maneuvers where conflict arose. In the experiments both vehicles had human drivers, but the remote vehicle's speed was regulated by cruise control (set to $30[\mathrm{mi} / \mathrm{hr}]=13.4[\mathrm{~m} / \mathrm{s}])$, which may be considered as a low level of automation. The ego vehicle started from standstill from the locations marked by magenta dots in panel (a) and it launched some time after the remote vehicle has passed a landmark (indicated by the red line). Thus, by varying the launching location and the launching time, a set of different initial conditions were explored. The ego vehicle's decision (and the corresponding act) on whether to merge ahead or behind the remote vehicle was made by the human driver. For each initial condition, multiple experiments were performed.

Both vehicles were equipped with GPS devices and V2X communication devices that allowed them to share their status (GPS-based position and speed) with a $10 \mathrm{~Hz}$ update rate using basic safety messages (BSMs) [30]. For intent sharing, the intended speed of the remote vehicle $(13.4[\mathrm{~m} / \mathrm{s}])$ was known by the driver of the ego vehicle. Correspondingly, when constructing the conflict chart in the $\left(r_{1}, r_{2}\right)$-plane in Fig. 12(a), we used velocity limits $\underline{v}_{1}=13[\mathrm{~m} / \mathrm{s}]$ and $\bar{v}_{1}=14[\mathrm{~m} / \mathrm{s}]$ (corresponding to the speed error of the cruise control) and $v_{\min , 2}=0$ and $v_{\max , 2}=20[\mathrm{~m} / \mathrm{s}]$ (the speed limits of the ego vehicle), while the other parameters are the same as in Table I. In this figure only the conflict chart of merge ahead is shown (cf. Figs. 3(d) and 6(d)) as in the experiments, due to the standstill initial condition, the ego vehicle could always merge behind the remote vehicle without conflict.

The initial condition for each experiment is displayed on the safety chart in Fig. 12(a). Green and blue circles correspond to non-conflicting merge ahead and merge behind runs, respectively, while magenta crosses indicate runs with conflict. Observe that the theoretical boundaries match the data well. Most non-conflicting merge ahead cases are located in the $\overline{\mathcal{P}}_{\mathrm{g}}$ region, while none of them appear in the $\overline{\mathcal{P}}_{\mathrm{r}}$ region. On the other hand, most conflicting cases are located in the $\overline{\mathcal{P}}_{\mathrm{y}}$ and $\overline{\mathcal{P}}_{\mathrm{r}}$ regions. These correspond to the human driver attempting to merge ahead based on an incorrect assessment of the situation. We remark that if rolling and air resistances were included in the ego vehicle's model, one would expect a slightly smaller green region and a slightly larger red region. We leave the quantification of this difference for our future work as this requires additional numerical tools.

Fig. 12(b)-(c) show the vehicles' motion data corresponding to point A (no conflict) and point B (conflict) in Fig. 12(a). In both cases, the ego vehicle decides to merge ahead as shown by the similar speed and acceleration profiles within the first 3.5 seconds in Fig. 12(c) and (d). However, in the conflict chart point A is located in the $\overline{\mathcal{P}}_{\mathrm{g}}$ region while point $\mathrm{B}$ is in the $\overline{\mathcal{P}}_{\mathrm{r}}$ region where a non-conflicting merge ahead is not possible. Correspondingly, as revealed by the speed and acceleration profiles after 3.5 seconds, the ego vehicle successfully merges ahead in case A but runs into a conflict in case B. In the latter case, the ego vehicle reduces its speed and acceleration and eventually merges behind the remote vehicle. The conflict is illuminated in Fig. 12(b) by red shading, where the ego vehicle (solid magenta curve) appears in the conflict zone at the same 

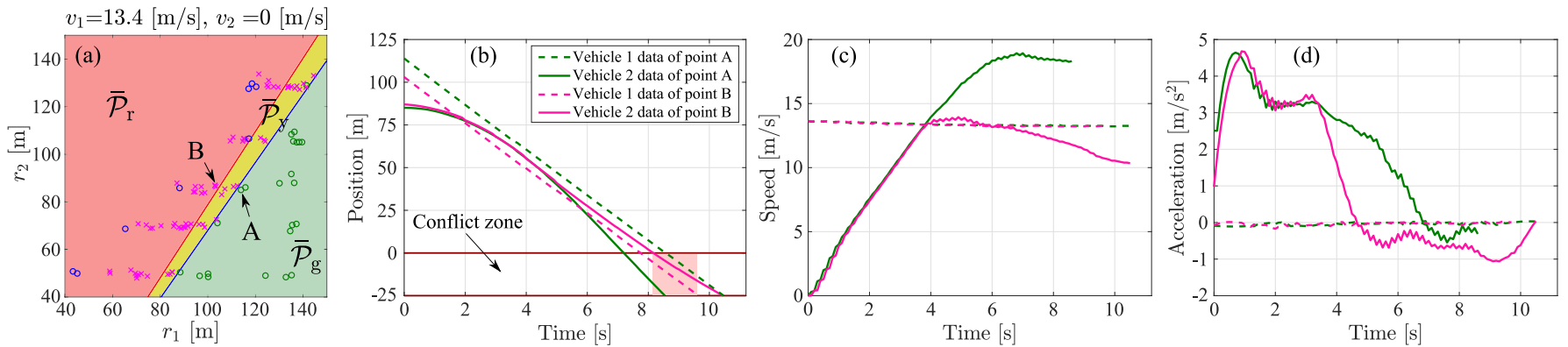

Fig. 12. (a) Experimental data (initial conditions of both vehicles) superimposed on the conflict chart. Green circles mark non-conflicting merges ahead, blue circles denote non-conflicting merges behind and magenta crosses correspond to conflicts. (b)-(d) Position, speed and acceleration profiles of both vehicles corresponding to the initial conditions of points A and B. The red shaded region in panel (b) highlights the time interval where both vehicles are inside the conflict zone in case B.
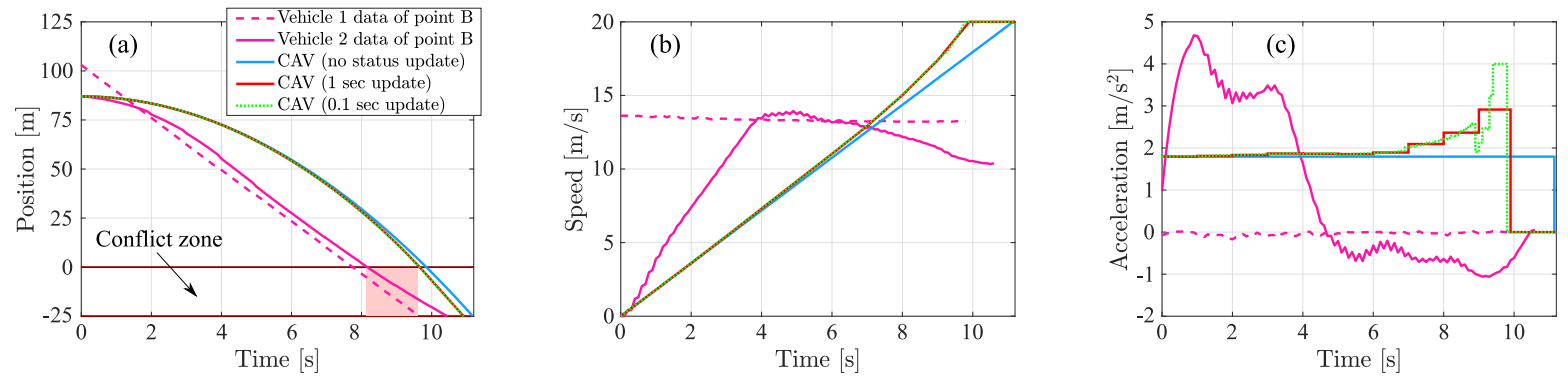

Fig. 13. Simulation results with the initial condition of point B in Fig. 12(a). Position, speed, and acceleration profiles of the vehicles are plotted in panels (a), (b), and (c), respectively for the experimental run and when the CAV uses the controller $(35,37,38,39)$ with different status update rates as indicated. The red shaded region in panel (a) highlights the time interval where both vehicles are inside the conflict zone in case B.

time as the remote vehicle (dashed magenta curve).

The ego vehicle was able to prevent conflict in case A but failed to do so in case B due to the inability of the human driver to accurately assess the remote vehicle's state and intent and to make an informed decision. If the ego vehicle was a connected automated vehicle, it could utilize V2X connectivity to obtain accurate information about the motion of the remote vehicle. Then, with the help of conflict analysis, the CAV could make a decision to prevent the conflict and execute the corresponding maneuver using the controller designed above. To demonstrate this, we use the initial condition of the point $\mathrm{B}$ in Fig. 12(a) and show simulation results for a CAV equipped with the controller (35) in Fig. 13.

Since $x(0) \notin \overline{\mathcal{P}}_{\mathrm{g}}$, according to (23), the CAV decides to merge behind the remote vehicle and applies (37)-(39). The position profiles in Fig. 13(a) show that the CAV enters the conflict zone just after the remote vehicle exits. The velocity and acceleration profiles in Fig. 13(b) and (c) highlight the differences caused by different V2X packet update rates. When no status updates are utilized (blue curves) the ego vehicle maintains a constant acceleration until it reaches the maximum speed. With status updates the control commands is updated every second (red curves) or every 0.1 seconds (green curves) yielding velocity and acceleration profiles which allow the $\mathrm{CAV}$ to execute the maneuver faster as can be observed in Fig. 13(a).

\section{B. Simulations using real highway data}

To further evaluate the efficiency of the decision making and control algorithms, we utilize data collected on US-23 near
TABLE II

MANEUVER EXeCUTION TIME OF THE CAV IN FIg. 14.

\begin{tabular}{|c|c|}
\hline & Execution time \\
\hline No status update & $13.58[\mathrm{~s}]$ \\
\hline Status update every 1 s & $10.45[\mathrm{~s}]$ \\
\hline Status update every 0.1 s & $10.31[\mathrm{~s}]$ \\
\hline Intent + Status & $7.07[\mathrm{~s}]$ \\
\hline
\end{tabular}

Ann Arbor, Michigan for the remote vehicle; see Fig. 2(a). The remote vehicle's position, velocity, and acceleration are shown by black curves in Fig. 14(a), (b), and (c), respectively. At the initial time the remote vehicle is 201.57 meters from the conflict zone traveling at a speed of $22.63[\mathrm{~m} / \mathrm{s}]$. At the same time, the ego vehicle (CAV) is placed at the on-ramp 210 meters from the conflict zone traveling with speed of 25 [m/s]. This yields $x(0) \in \mathcal{P}_{\mathrm{y}} \cap Q_{\mathrm{g}}$ and, according to (23), the CAV decides to merge behind the remote vehicle while using the controller (35) with (37)-(39). In Fig. 14, the time profiles of the CAV are shown for different status update rates; see blue, red and green curves. Notice that the time needed for the CAV to execute the maneuver (i.e., execution time) decreases when status packets are received more frequently; as shown in Table II. Less frequent status updates yield longer execution times due to the conservative prediction of remote vehicle's future motion.

When intent information is available, the execution times can be further reduced. For example, extracting the bounds $\underline{v}_{1}=21[\mathrm{~m} / \mathrm{s}], \bar{v}_{1}=27[\mathrm{~m} / \mathrm{s}], \underline{a}_{1}=-1\left[\mathrm{~m} / \mathrm{s}^{2}\right], \bar{a}_{1}=1\left[\mathrm{~m} / \mathrm{s}^{2}\right]$ from the velocity and acceleration data of the remote vehicle, yields $x(0) \in \overline{\mathcal{P}}_{\mathrm{g}} \cap \bar{Q}_{\mathrm{g}}$, that is, the CAV decides to merge ahead using the controller (35) with (36); see the purple 

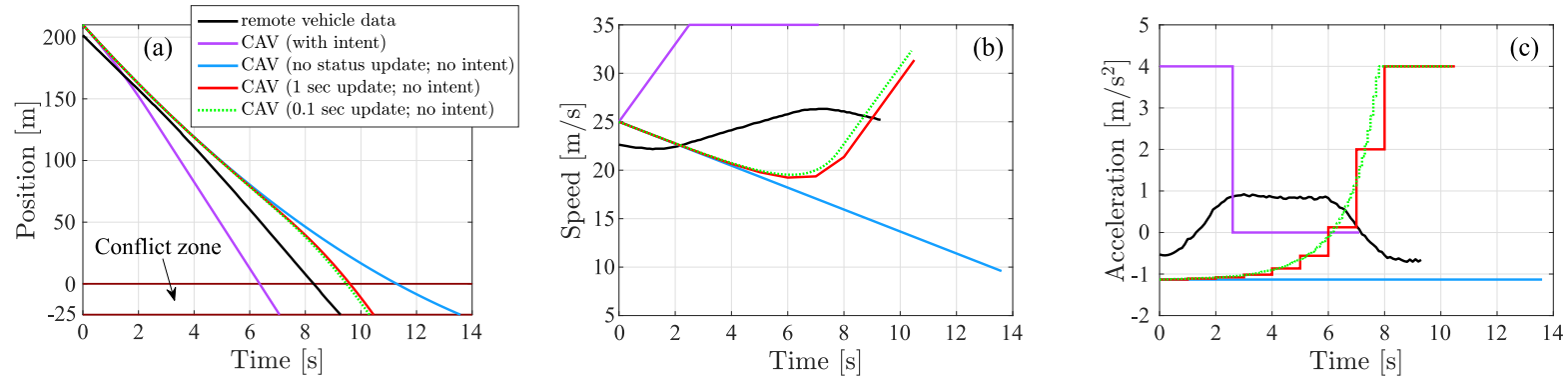

Fig. 14. Simulation results when a CAV utilizes traffic data received from the remote vehicle and applies the controller $(35,36,37,38,39)$ with different status update rates and intent as indicated. Position, speed, and acceleration profiles of the vehicles are plotted in panels (a), (b), and (c), respectively. Observe that with intent information the CAV's decision changes from merge behind to merge ahead.
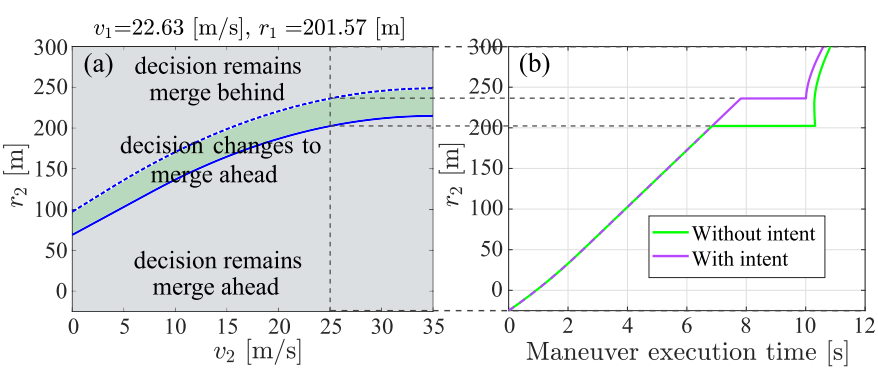

Fig. 15. (a) Change of decision chart corresponding to the initial condition of the remote vehicle in Fig. 14. (b) Time needed for the CAV to execute the maneuver when choosing the initial condition along the dashed vertical line.

curves in Fig. 14. This decision change leads to a significant improvement of the execution time as shown in Table II.

Figure 15(a) depicts the domain in state space where the CAV changes its decision from merge behind to merge ahead if intent information becomes available. Considering the initial position $r_{2}(0) \in[-s, 300][\mathrm{m}]$ and initial speed $v_{2}(0)=25[\mathrm{~m} / \mathrm{s}]$ for the CAV (marked by the vertical dashed line in Fig. 15(a)), we simulated the CAV without and with intent while using the controller (35)-(39). The corresponding maneuver execution times are shown in Fig. 15(b) for status update rate $10 \mathrm{~Hz}$. Observe that the execution time is significantly reduced in the domain where the decision changes due to intent. Even in the domain where the decision merge behind remains unchanged, having intent information still improves the time efficiency of the controller.

\section{CONCLUSION}

In this paper, we established the concept of conflict analysis in order to help vehicles with different levels of automation and cooperation to prevent conflicts. We constructed conflict charts that allowed connected automated vehicles to make better decisions while utilizing status and intent messages received from remote vehicles via V2X communication. We also determined the communication range required to guarantee the existence of conflict-free maneuvers. We demonstrated that increasing the frequency of status updates can benefit the time efficiency of the ego vehicle. Moreover, we showed that sharing intent information that bounds the future speed and acceleration of remote vehicles leads to less conservatism in the decision making of the ego vehicle. Intent messages by remote vehicles also yield significant improvements in the time efficiency of the ego vehicle and such improvements cannot be replicated by merely sharing current status information more frequently. The results were demonstrated experimentally using real vehicles on a test track and by numerical simulations using real highway data.

As our future work, the proposed conflict analysis framework will be extended to more complex scenarios. For example, we plan to study conflicts between multiple vehicles, by defining pairwise conflicts between the ego vehicle and individual target vehicles using formal logic and combining the pairwise conflict charts. We will also implement more detailed vehicle dynamics models to account for the effects of rolling resistance, air resistance and time delays on conflict analysis. Since analytical calculation will become unfeasible, we will develop numerical tools for conflict analysis. We will also consider more sophisticated controller design which takes into account the passenger comfort and energy consumption. Other extensions of this work include considering more realistic intent information where velocity and acceleration ranges may be time-varying, and investigating higher levels of cooperation between vehicles.

\section{APPENDIX A \\ ProOF OF $P \vee Q \Longleftrightarrow \neg C$}

To prove $P \vee Q \Rightarrow \neg C$, we only need to prove that $(P \Rightarrow \neg C) \wedge(Q \Rightarrow \neg C)$. Let us first focus on the proposition $P$. Let $t_{P}$ be the time such that $r_{1}\left(t_{P}\right)=0$ and $r_{2}\left(t_{P}\right)<-s$ hold. Since $r_{1}(t)$ is monotonously decreasing, and $r_{2}(t)$ is non-increasing with respect to $t$, the following statements hold:

$$
\begin{array}{ll}
\forall t<t_{P}, & r_{1}(t)>0, \\
\forall t \geq t_{P}, & r_{2}(t)<-s .
\end{array}
$$

Thus, $P \Rightarrow \neg C$. Similarly, for proposition $Q$, let $t_{Q}$ be the time such that $r_{1}\left(t_{Q}\right)=-s$ and $r_{2}\left(t_{Q}\right)>0$ hold. Then we have

$$
\begin{array}{ll}
\forall t>t_{Q}, & r_{1}(t)<-s, \\
\forall t \leq t_{Q}, & r_{2}(t)>0 .
\end{array}
$$

Thus, $Q \Rightarrow \neg C$. Therefore, $P \vee Q \Rightarrow \neg C$.

To prove $P \vee Q \Leftarrow \neg C$, we prove its contrapositive $(\neg P \wedge \neg Q) \Rightarrow C$. From (6), we have

$$
\begin{aligned}
& \neg P=\left\{\forall t, r_{1}(t) \neq 0 \vee r_{2}(t) \geq-s\right\}, \\
& \neg Q=\left\{\forall t, r_{1}(t) \neq-s \vee r_{2}(t) \leq 0\right\} .
\end{aligned}
$$


If $(\neg P \wedge \neg Q)=$ true, then both $\neg P=$ true and $\neg Q=$ true. Let $t_{P}$ and $t_{Q}$ be the times such that $r_{1}\left(t_{P}\right)=0$ and $r_{1}\left(t_{Q}\right)=-s$, respectively. Based on (44), $r_{2}\left(t_{P}\right) \geq-s$ and $r_{2}\left(t_{Q}\right) \leq 0$ must hold. Then we have $\neg P \Longrightarrow P_{1} \vee P_{2}$, where $P_{1}$ and $P_{2}$ are the propositions

$$
\begin{aligned}
& P_{1}:=\left\{\exists t_{P}, r_{1}\left(t_{P}\right)=0 \wedge r_{2}\left(t_{P}\right) \in[-s, 0]\right\}, \\
& P_{2}:=\left\{\exists t_{P}, r_{1}\left(t_{P}\right)=0 \wedge r_{2}\left(t_{P}\right) \in(0, \infty)\right\} .
\end{aligned}
$$

Note that here we divided $r_{2}\left(t_{P}\right) \geq-s$ into two cases: $r_{2}\left(t_{P}\right) \in[-s, 0]$ and $r_{2}\left(t_{P}\right) \in(0, \infty)$. Similarly, we obtain $\neg Q \Longrightarrow Q_{1} \vee Q_{2}$, where $Q_{1}$ and $Q_{2}$ are

$$
\begin{aligned}
& Q_{1}:=\left\{\exists t_{Q}, r_{1}\left(t_{Q}\right)=-s \wedge r_{2}\left(t_{Q}\right) \in[-s, 0]\right\}, \\
& Q_{2}:=\left\{\exists t_{Q}, r_{1}\left(t_{Q}\right)=-s \wedge r_{2}\left(t_{Q}\right) \in(-\infty,-s)\right\} .
\end{aligned}
$$

Therefore, $(\neg P \wedge \neg Q) \Longrightarrow\left(P_{1} \vee P_{2}\right) \wedge\left(Q_{1} \vee Q_{2}\right)$. On the other hand,

$$
\begin{aligned}
& \left(P_{1} \vee P_{2}\right) \wedge\left(Q_{1} \vee Q_{2}\right) \\
& =\left(P_{1} \wedge Q_{1}\right) \vee\left(P_{1} \wedge Q_{2}\right) \vee\left(P_{2} \wedge Q_{1}\right) \vee\left(P_{2} \wedge Q_{2}\right) .
\end{aligned}
$$

One can confirm that $\left(P_{2} \wedge Q_{2}\right) \Longrightarrow C$. Also, $P_{1} \Longrightarrow C$ and $Q_{1} \Longrightarrow C$. Thus, it follows from (47) that $(\neg P \wedge \neg Q) \Longrightarrow C$. These complete the proof.

\section{APPENDIX B}

\section{CONFLICT CHART BOUNDARIES}

Boundaries $p_{1}$ and $p_{2}$ in (14), (15), and (16) are given by

$$
\begin{aligned}
& r_{2}=p_{1}\left(r_{1}, v_{1}, v_{2}\right)=p\left(t_{\mathrm{p} 1}, v_{2}\right), \\
& r_{2}=p_{2}\left(r_{1}, v_{1}, v_{2}\right)=p\left(t_{\mathrm{p} 2}, v_{2}\right),
\end{aligned}
$$

where

$$
\begin{aligned}
& p\left(t_{\mathrm{p} 1}, v_{2}\right)= \\
& \begin{cases}t_{\mathrm{p} 1} v_{2}+\frac{1}{2} t_{\mathrm{p} 1}^{2} a_{\max , 2}-s & \text { if } v_{2} \leq v_{\max , 2}-t_{\mathrm{p} 1} a_{\max , 2}, \\
-\frac{\left(v_{\max , 2}-v_{2}\right)^{2}}{2 a_{\max , 2}}+t_{\mathrm{p} 1} v_{\max , 2}-s & \text { if } v_{2}>v_{\max , 2}-t_{\mathrm{p} 1} a_{\max , 2},\end{cases}
\end{aligned}
$$

with $t_{\mathrm{p} 1}=t_{\mathrm{p}}\left(a_{\max , 1}\right)$ and $t_{\mathrm{p} 2}=t_{\mathrm{p}}\left(a_{\min , 1}\right)$, where the function $t_{\mathrm{p}}(a)$ is given by the following three cases depending on the sign of its argument.

(i) For $a>0$,

$$
t_{\mathrm{p}}(a)= \begin{cases}\frac{\sqrt{v_{1}^{2}+2 a r_{1}}-v_{1}}{a} & \text { if } r_{1} \leq \frac{v_{\max , 1}^{2}-v_{1}^{2}}{2 a}, \\ \frac{v_{\max , 1}-v_{1}}{a}+\frac{r_{1}-\frac{v_{\max , 1}^{2}-v_{1}^{2}}{2 a}}{v_{\max , 1}} & \text { if } r_{1}>\frac{v_{\max , 1}^{2}-v_{1}^{2}}{2 a} .\end{cases}
$$

(ii) For $a=0$,

$$
t_{\mathrm{p}}(a)=\frac{r_{1}}{v_{1}}
$$

(iii) For $a<0$,

$$
t_{\mathrm{p}}(a)= \begin{cases}-\frac{v_{1}-\sqrt{v_{1}^{2}+2 a r_{1}}}{a} & \text { if } r_{1} \leq-\frac{v_{1}^{2}-v_{\min , 1}^{2}}{2 a}, \\ -\frac{v_{1}-v_{\min , 1}}{a}+\frac{r_{1}+\frac{v_{1}^{2}-v_{\min , 1}^{2}}{2 a}}{v_{\min , 1}} & \text { if } r_{1}>-\frac{v_{1}^{2}-v_{\min , 1}^{2}}{2 a} .\end{cases}
$$

Note that $t_{\mathrm{p} 1}$ and $t_{\mathrm{p} 2}$ are the time needed for the remote vehicle to reach conflict zone, with $u_{1}(t) \equiv a_{\max , 1}$ and $u_{1}(t) \equiv a_{\min , 1}, \quad$ respectively. Thus, the relation $t_{\mathrm{p} 2} \geq t_{\mathrm{p} 1}$ always holds, and therefore, we have $p_{2}\left(r_{1}, v_{1}, v_{2}\right) \geq p_{1}\left(r_{1}, v_{1}, v_{2}\right)$.

Boundaries $q_{1}$ and $q_{2}$ in (17), (18), and (19) are given by

$$
\begin{aligned}
& r_{2}=q_{1}\left(r_{1}, v_{1}, v_{2}\right)=q\left(t_{\mathrm{q} 1}, v_{2}\right), \\
& r_{2}=q_{2}\left(r_{1}, v_{1}, v_{2}\right)=q\left(t_{\mathrm{q} 2}, v_{2}\right),
\end{aligned}
$$

where

$$
q\left(t_{\mathrm{q} 1}, v_{2}\right)= \begin{cases}t_{\mathrm{q} 1} v_{2}+\frac{1}{2} t_{\mathrm{q} 1}^{2} a_{\min , 2} & \text { if } v_{2} \geq-t_{\mathrm{q} 1} a_{\min , 2}, \\ -\frac{v_{2}^{2}}{2 a_{\min , 2}} & \text { if } v_{2}<-t_{\mathrm{q} 1} a_{\min , 2},\end{cases}
$$

with $t_{\mathrm{q} 1}=t_{\mathrm{q}}\left(a_{\min , 1}\right)$ and $t_{\mathrm{q} 2}=t_{\mathrm{q}}\left(a_{\max , 1}\right)$, where the function $t_{\mathrm{q}}(a)$ is defined below.

(i) For $a>0$,

$t_{\mathrm{q}}(a)= \begin{cases}\frac{\sqrt{v_{1}^{2}+2 a\left(r_{1}+s\right)}-v_{1}}{a} & \text { if } r_{1} \leq \frac{v_{\max , 1}^{2}-v_{1}^{2}}{2 a}-s, \\ \frac{v_{\max , 1}-v_{1}}{a}+\frac{r_{1}+s-\frac{v_{\max , 1}^{2}-v_{1}^{2}}{2 a}}{v_{\max , 1}} & \text { if } r_{1}>\frac{v_{\max , 1}^{2}-v_{1}^{2}}{2 a}-s .\end{cases}$

(ii) For $a=0$,

$$
t_{\mathrm{q}}(a)=\frac{r_{1}+s}{v_{1}}
$$

(iii) For $a<0$,

$$
t_{\mathrm{q}}(a)= \begin{cases}-\frac{v_{1}-\sqrt{v_{1}^{2}+2 a\left(r_{1}+s\right)}}{a} & \text { if } r_{1} \leq-\frac{v_{1}^{2}-v_{\min , 1}^{2}}{2 a}-s, \\ -\frac{v_{1}-v_{\min , 1}}{a}+\frac{r_{1}+s+\frac{v_{1}^{2}-v_{\min , 1}^{2}}{2 a}}{v_{\min , 1}} & \text { if } r_{1}>-\frac{v_{1}^{2}-v_{\min , 1}^{2}}{2 a}-s .\end{cases}
$$

We remark that in the above equations $a_{\max , 2}>0, a_{\min , 2}<0$, and $0<v_{\min , 1}<v_{\max , 1}$ hold by definition, and thus, the fractions are well-defined.

\section{APPENDIX C \\ PROOF OF THEOREM 1}

Notice that boundary $q_{1}$ is upper bounded by $r_{2}=f\left(v_{2}\right)=-v_{2}^{2} /\left(2 a_{\min , 2}\right)$. Thus, to prove that $x(0) \in \mathcal{P}_{\mathrm{g}} \cup \mathcal{Q}_{\mathrm{g}}$ holds for $r_{1} \geq r_{1}^{*}, \quad$ it is sufficient to show that $r_{1} \geq r_{1}^{*} \Rightarrow p_{1}\left(r_{1}, v_{1}, v_{2}\right)>f\left(v_{2}\right)$, $\forall v_{1} \in\left[v_{\min , 1}, v_{\max , 1}\right], \forall v_{2} \in\left[0, v_{\max , 2}\right]$.

Let us define

$$
\delta\left(r_{1}, v_{1}, v_{2}\right):=p_{1}\left(r_{1}, v_{1}, v_{2}\right)-f\left(v_{2}\right) .
$$

By calculating $\frac{\partial \delta}{\partial v_{2}}\left(r_{1}, v_{1}, v_{2}\right)$, one may show that $\delta$ first increases and then decreases with respect to $v_{2}$ on $\left[0, v_{\max , 2}\right]$. That is, $\delta$ takes minimum value at $v_{2}=0$ or $v_{2}=v_{\max , 2}$.

Now consider the inequalities

$$
\begin{aligned}
\delta\left(r_{1}, v_{1}, 0\right)>0 & \Longleftrightarrow r_{1}>g_{1}\left(v_{1}\right), \\
\delta\left(r_{1}, v_{1}, v_{\max , 2}\right)>0 & \Longleftrightarrow r_{1}>g_{2}\left(v_{1}\right),
\end{aligned}
$$

where $g_{1}$ and $g_{2}$ are functions of $v_{1}$. One can confirm that the $\underline{r}_{1}$ and $\bar{r}_{1}$ given in (21) correspond to the maximum values of $g_{1}$ and $g_{2}$ on $v_{1} \in\left[v_{\min , 1}, v_{\max , 1}\right]$. Thus, if $r_{1} \geq r_{1}^{*}=\max \left\{\underline{r}_{1}, \bar{r}_{1}\right\}$, then both (61) and (62) hold independent of $v_{1}$. This yields that $\delta\left(r_{1}, v_{1}, v_{2}\right)>0$, i.e., $p_{1}\left(r_{1}, v_{1}, v_{2}\right)>f\left(v_{2}\right), \forall v_{1} \in\left[v_{\min , 1}, v_{\max , 1}\right], \forall v_{2} \in\left[0, v_{\max , 2}\right]$. 


\section{APPENDIX D \\ Proof of THEOREM 2}

To prove (30) and (31), let us first prove the following two statements under vehicle 1 's intent $v_{1} \in\left[\underline{v}_{1}, \bar{v}_{1}\right]$ and $u_{1} \in\left[\underline{a}_{1}, \bar{a}_{1}\right]:$

$$
\begin{aligned}
& \bar{p}_{1}\left(r_{1}, v_{1}, v_{2}\right) \geq p_{1}\left(r_{1}, v_{1}, v_{2}\right), \\
& \bar{p}_{2}\left(r_{1}, v_{1}, v_{2}\right) \leq p_{2}\left(r_{1}, v_{1}, v_{2}\right), \\
& \forall r_{1} \in(0, \infty), v_{1} \in\left[\underline{v}_{1}, \bar{v}_{1}\right], v_{2} \in\left[0, v_{\max , 2}\right],
\end{aligned}
$$

and

$$
\begin{aligned}
& \bar{q}_{1}\left(r_{1}, v_{1}, v_{2}\right) \leq p_{1}\left(r_{1}, v_{1}, v_{2}\right) \\
& \bar{q}_{2}\left(r_{1}, v_{1}, v_{2}\right) \geq q_{2}\left(r_{1}, v_{1}, v_{2}\right), \\
& \forall r_{1} \in[-s, \infty), v_{1} \in\left[\underline{v}_{1}, \bar{v}_{1}\right], v_{2} \in\left[0, v_{\max , 2}\right] .
\end{aligned}
$$

Using (51)-(53) and (57)-(59), we calculate $\bar{t}_{p 1}, \bar{t}_{p 2}, \bar{t}_{q 1}$, and $\bar{t}_{q 2}$ with the remote vehicle's velocity and acceleration limits given by its intent. Since these limits never exceed the range $\left[v_{\min , 1}, v_{\max , 1}\right]$ and $\left[a_{\min , 1}, a_{\max , 1}\right]$ (cf. Definition 2), one can confirm that $\bar{t}_{p 1} \geq t_{p 1}, \bar{t}_{p 2} \leq t_{p 2}, \bar{t}_{q 1} \leq t_{q 1}$ and $\bar{t}_{q 2} \geq t_{q 2}$. Moreover, from (50) and (56), the function $p$ monotonically increases with respect to $t_{p 1}$ and $t_{p 2}$, and $q$ monotonically increases with respect to $t_{q 1}$ and $t_{q 2}$. Then, (63) and (64) follow from (48)-(49) and (54)-(55).

Finally, (30) is proved by combining (63) with (14)-(16) and (24)-(26), and (31) is proved by combining (64) with (17)-(19) and (27)-(29).

\section{REFERENCES}

[1] SAE J3216, "Taxonomy and Definitions for Terms Related to Cooperative Driving Automation for On-Road Motor Vehicles," SAE International, Tech. Rep., 2020.

[2] T. Ersal, I. Kolmanovsky, N. Masoud, N. Ozay, J. Scruggs, R. Vasudevan, and G. Orosz, "Connected and automated road vehicles: state of the art and future challenges," Vehicle System Dynamics, vol. 58, no. 5, pp. 672-704, 2020.

[3] K. Abboud, H. A. Omar, and W. Zhuang, "Interworking of DSRC and cellular network technologies for V2X communications: A survey," IEEE Transactions on Vehicular Technology, vol. 65, no. 12, pp. 94579470, 2016.

[4] J. I. Ge, S. S. Avedisov, C. R. He, W. B. Qin, M. Sadeghpour, and G. Orosz, "Experimental validation of connected automated vehicle design among human-driven vehicles," Transportation Research Part C, vol. 91, pp. 335-352, 2018.

[5] S. S. Avedisov, G. Bansal, and G. Orosz, "Impacts of connected automated vehicles on freeway traffic patterns at different penetration levels," IEEE Transactions on Intelligent Transportation Systems, 2021. [Online]. Available: https://doi.org/10.1109/TITS.2020.3043323

[6] L. Hobert, A. Festag, I. Llatser, L. Altomare, F. Visintainer, and A. Kovacs, "Enhancements of V2X communication in support of cooperative autonomous driving," IEEE Communications Magazine, vol. 53, no. 12, pp. 64-70, 2015.

[7] B. Lehmann, H. J. Günther, and L. Wolf, "A generic approach towards maneuver coordination for automated vehicles," in 21st IEEE International Conference on Intelligent Transportation Systems (ITSC), 2018, pp. 3333-3339.

[8] I. Llatser, T. Michalke, M. Dolgov, F. Wildschütte, and H. Fuchs, "Cooperative automated driving use cases for 5G V2X communication," in 2nd IEEE 5G World Forum (5GWF), 2019, pp. 120-125.

[9] A. Correa, S. Maerivoet, E. Mintsis, A. Wijbenga, M. Sepulcre, M. Rondinone, J. Schindler, and J. Gozalvez, "Management of transitions of control in mixed traffic with automated vehicles," in 16th International Conference on Intelligent Transportation Systems Telecommunications (ITST), 2018, pp. 1-7.

[10] A. I. M. Medina, N. van de Wouw, and H. Nijmeijer, "Cooperative intersection control based on virtual platooning," IEEE Transactions on Intelligent Transportation Systems, vol. 19, no. 6, pp. 1727-1740, 2021.
[11] C. Liu, C.-W. Lin, S. Shiraishi, and M. Tomizuka, "Distributed conflict resolution for connected autonomous vehicles," IEEE Transactions on Intelligent Vehicles, vol. 3, no. 1, pp. 18-29, 2020.

[12] R. Hult, M. Zanon, S. Gros, H. Wymeersch, and P. Falcone, "Optimisation-based coordination of connected, automated vehicles at intersections," Vehicle System Dynamics, vol. 58, no. 5, pp. 726-747, 2020.

[13] J. Rios-Torres and A. A. Malikopoulos, "A survey on the coordination of connected and automated vehicles at intersections and merging at highway on-ramps," IEEE Transactions on Intelligent Transportation Systems, vol. 18, no. 5, pp. 1066-1077, 2016.

[14] I. B. Viana, H. Kanchwala, and N. Aouf, "Cooperative trajectory planning for autonomous driving using nonlinear model predictive control," in IEEE International Conference on Connected Vehicles and Expo (ICCVE), 2019, pp. 1-6.

[15] M. R. Hafner and D. Del Vecchio, "Computational tools for the safety control of a class of piecewise continuous systems with imperfect information on a partial order," SIAM Journal on Control and Optimization, vol. 49, no. 6, pp. 2463-2493, 2011.

[16] R. Kianfar, P. Falcone, and J. Fredriksson, "Safety verification of automated driving systems," IEEE Intelligent Transportation Systems Magazine, vol. 5, no. 4, pp. 73-86, 2013.

[17] M. R. Hafner, D. Cunningham, L. Caminiti, and D. Del Vecchio, "Cooperative collision avoidance at intersections: Algorithms and experiments," IEEE Transactions on Intelligent Transportation Systems, vol. 14, no. 3, pp. 1162-1175, 2013.

[18] S. Bansal, M. Chen, S. Herbert, and C. J. Tomlin, "Hamilton-Jacobi reachability: a brief overview and recent advances," in the 56th IEEE Conference on Decision and Control, Melbourne, Australia, 2017, pp. 2242-2253.

[19] Z. Lin, L. Castano, E. Mortimer, and H. Xu, "Fast 3D collision avoidance algorithm for fixed wing uas," Journal of Intelligent and Robotic Systems, vol. 97, no. 3, pp. 577-604, 2020.

[20] J. N. Yasin, S. A. S. Mohamed, M. Haghbayan, J. Heikkonen, H. Tenhunen, and J. Plosila, "Unmanned aerial vehicles (UAVs): Collision avoidance systems and approaches," IEEE Access, vol. 8, pp. 105139 $105155,2020$.

[21] A. Bajcsy, S. L. Herbert, D. Fridovich-Keil, J. F. Fisac, S. Deglurkar, A. Dragan, and C. Tomlin, "A scalable framework for real-time multirobot, multi-human collision avoidance," 2019 International Conference on Robotics and Automation (ICRA), pp. 936-943, 2019.

[22] B. M. Albaba and Y. Yildiz, "Modeling cyber-physical human systems via an interplay between reinforcement learning and game theory," Annual Reviews in Control, vol. 48, pp. 1-21, 2019.

[23] M. Karimi, C. Roncoli, C. Alecsandru, and M. Papageorgiou, "Cooperative merging control via trajectory optimization in mixed vehicular traffic," Transportation Research Part C: Emerging Technologies, vol. 116, p. $102663,2020$.

[24] Z. Sun, T. Huang, and P. Zhang, "Cooperative decision-making for mixed traffic: A ramp merging example," Transportation Research Part C: Emerging Technologies, vol. 120, p. 102764, 2020.

[25] J. Guo, S. Cheng, and Y. Liu, "Optimal control of connected and automated vehicles at roundabouts: an investigation in a mixed-traffic environment," IFAC-PapersOnLine, vol. 51, no. 9, pp. 73-78, 2018, 15th IFAC Symposium on Control in Transportation Systems CTS 2018.

[26] A. Kondyli and L. Elefteriadou, "Modeling driver behavior at freewayramp merges," Transportation Research Record, vol. 2249, no. 1, pp. 29-37, 2011.

[27] D. Zhou, Z. Ma, and J. Sun, "Autonomous vehicles' turning motion planning for conflict areas at mixed-flow intersections," IEEE Transactions on Intelligent Vehicles, vol. 5, no. 2, pp. 204-216, 2020.

[28] Y. Zhang, Q. Lin, J. Wang, S. Verwer, and J. M. Dolan, "Lane-change intention estimation for car-following control in autonomous driving," IEEE Transactions on Intelligent Vehicles, vol. 3, no. 3, pp. 276-286, 2018.

[29] H. M. Wang, T. G. Molnár, S. S. Avedisov, A. H. Sakr, O. Altintas, and G. Orosz, "Conflict analysis for cooperative merging using V2X communication," in 31st IEEE Intelligent Vehicles Symposium, Las Vegas, USA, 2020, pp. 1538-1543.

[30] SAE J2735, "Dedicated Short Range Communications (DSRC) Message Set Dictionary Set," SAE International, Tech. Rep., 2016. 

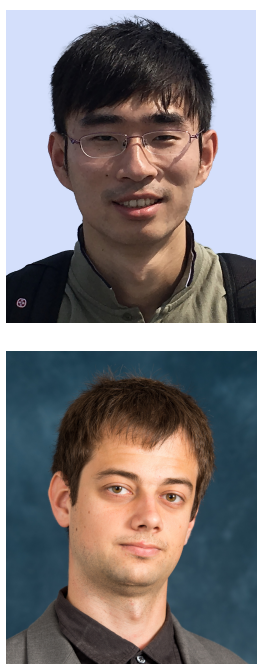

Hao M. Wang received the B.Eng. degree in mechanical and aerospace engineering from Nagoya University, Nagoya, Japan, in 2018. He is currently working toward the Ph.D. degree in mechanical engineering at the University of Michigan, Ann Arbor, USA. His research interests include dynamics and control of connected and automated vehicles.

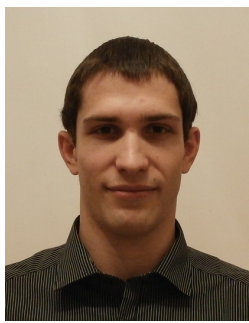

Tamás G. Molnár received his B.Sc. degree in Mechatronics Engineering, M.Sc. and Ph.D. degrees in Mechanical Engineering from the Budapest University of Technology and Economics, Budapest, Hungary, in 2013, 2015 and 2018. He held postdoctoral position at the University of Michigan, Ann Arbor between 2018 and 2020. He is currently a postdoctoral fellow at the California Institute of Technology, Pasadena. His research interests include nonlinear dynamics and control, safety-critical control, and time delay systems.

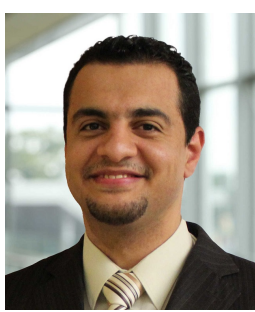

Ahmed Hamdi Sakr received the Ph.D. degree in Electrical and Computer Engineering from the University of Manitoba, Winnipeg, MB, Canada, in 2017. Currently, he is an Assistant Professor in the Department of Electrical and Computer Engineering at University of Windsor, Canada. Prior to that, he was a Principal Researcher at Toyota Motor North America R\&D where he was leading several research initiatives on the development of cooperative and automated driving functionalities. He serves as an Associate Editor for the IEEE Transactions on Vehicular Technology and a Track Co-chair for the IEEE Vehicular Technology Conference and IEEE Vehicular Networking Conference. His research interests include connected and autonomous vehicles, wireless and vehicular networks, Internet of Things (IoT), signal processing, and machine learning.

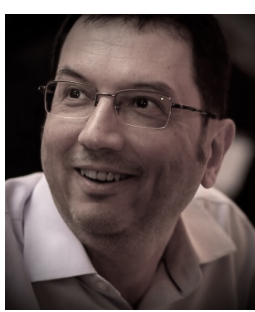

Onur Altintas received his Ph.D. degree in Electronics Engineering from The University of Tokyo. He has been with the Toyota Group since 1999 in various roles in New Jersey, Tokyo and California. $\mathrm{He}$ is currently the InfoTech Labs Fellow and Senior Executive Engineer at Toyota Motor North America R\&D, InfoTech Labs. He has been the co-founder and general co-chair of the IEEE Vehicular Networking Conference (IEEE VNC) since 2009. He serves as an associate editor for IEEE ITS Magazine, IEEE Vehicular Technology Magazine and IEEE Transactions on Intelligent Vehicles. He is an IEEE Vehicular Technology Society Distinguished Lecturer.

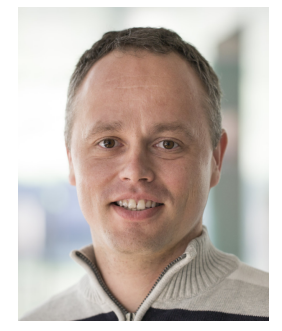

Gábor Orosz received the M.Sc. degree in Engineering Physics from the Budapest University of Technology, Hungary, in 2002 and the Ph.D. degree in Engineering Mathematics from University of Bristol, UK, in 2006. He held postdoctoral positions at the University of Exeter, UK, and at the University of California, Santa Barbara. In 2010, he joined the University of Michigan, Ann Arbor where he is currently an Associate Professor in Mechanical Engineering and in Civil and Environmental Engineering. His research interests include nonlinear dynamics and control, time delay systems, and machine learning with applications to connected and automated vehicles, traffic flow, and biological networks. 